\title{
Metformin alleviates inflammation through suppressing FASN- dependent palmitoylation of Akt
}

\author{
Wenfang Xiong ${ }^{1,4}$, Kuo-Yang Sun ${ }^{2,4}$, Yan Zhu ${ }^{1}$, Xiaoqi Zhang ${ }^{1 凶}$, Yi-Hua Zhou ${ }^{3 凶}$ and Xiaoping Zou (D) ${ }^{1 凶}$
}

(c) The Author(s) 2021

Metformin, traditionally regarded as a hypoglycemic drug, has been studied in other various fields including inflammation. The specific mechanism of metformin's effect on immune cells remains unclear. Herein, it is verified that LPS-induced macrophages are characterized by enhanced endogenous fatty acid synthesis and the inhibition of fatty acid synthase (FASN) downregulates proinflammatory responses. We further show that metformin could suppress such elevation of FASN as well as proinflammatory activation in macrophages. In vivo, metformin treatment ameliorates dextran sulfate sodium (DSS)-induced colitis through impairing proinflammatory activation of colonic lamina propria mononuclear cells (LPMCs). The reduction of FASN by metformin hinders Akt palmitoylation, which further disturbs Akt membrane attachment and its phosphorylation. Metformin-mediated suppression of FASN/Akt pathway and its downstream MAPK signaling contributes to its anti-inflammatory role in macrophages. From the perspective of immunometabolism, our work points towards metformin utilization as an effective and potential intervention against macrophages-involved inflammatory diseases.

Cell Death and Disease (2021)12:934; https://doi.org/10.1038/s41419-021-04235-0

\section{INTRODUCTION}

Macrophages display functional plasticity [1]. In recent years, researchers have been recognizing that activation, differentiation, and polarization of macrophages can trigger distinct changes in intracellular metabolic pathways (metabolic reprograming), which in turn contribute to shaping the immune responses and immune cell fate $[2,3]$. For example, proinflammatory macrophages possess enhanced glycolysis, whereas IL-4-induced macrophages rely on mitochondrial oxidative phosphorylation (OXPHOS) [4, 5]. Furthermore, several studies have also revealed that proinflammatory stimuli upregulate de novo fatty acid synthesis in macrophages [6]. Though a number of studies have indicated that exogenous fatty acid activates TLR related inflammatory signaling pathway in immune cells $[7,8]$, the endogenous fatty acid has been being evidenced to regulate inflammation and immune as well $[9,10]$. Intracellular palmitic acid synthesized by fatty acid synthase (FASN) can be attached to protein as a modification called palmitoylation [11-14]. Palmitoylation could enhance the hydrophobicity of protein and further influence protein trafficking and functions, which bridges fatty acid metabolism and other cellular activities including inflammatory responses. It has been reported that palmitoylation of MYD88 and NOD participated in regulating inflammatory responses $[11,12,15]$. Warburg effect as a cancer target $[16]$, we inferred that fatty acid synthesis was a novel target pathway to suppress proinflammatory activation of macrophages.
Metformin, as a first-line drug for type 2 diabetes mellitus, reduces blood glucose partly via activating AMP-activated protein kinase (AMPK) [17]. In addition, several investigations have reported that the drug improves liver triglyceride accumulation, dyslipidemia, and atherosclerosis [18-20]. In the past years it has also been suggested that metformin elicits anti-inflammatory action via acting on macrophages directly [21-23]. Owing to the importance of fatty acid synthesis to proinflammatory activation in macrophages as well as the possible effect of metformin on lipid metabolism, we hypothesized that metformin could inhibit proinflammatory activation of macrophages via reducing their fatty acid synthesis, which further alleviated inflammation.

In our study, we show that proinflammatory macrophages are characterized by increased endogenous fatty acid synthesis and the inhibition of FASN downregulates proinflammatory responses of macrophages. We further identify that metformin could suppress LPS-induced elevation of FASN and proinflammatory responses in macrophages as well. The inhibition of FASN by metformin reduces Akt palmitoylation, which is closely associated with downregulated Akt membrane recruitment and phosphorylation. Metformin-mediated suppression of FASN/Akt pathway and its downstream MAPK signaling contributes to its antiinflammatory role in macrophages. Consistent with in vitro results, metformin treatment ameliorates dextran sulfate sodium (DSS)induced colitis through impairing proinflammatory activation of colonic lamina propria mononuclear cells (LPMCs).

\footnotetext{
'Department of Gastroenterology, Nanjing Drum Tower Hospital, The Affiliated Hospital of Nanjing University Medical School, 321 Zhongshan Road, Nanjing 210008 Jiangsu, PR China. ${ }^{2}$ State Key Laboratory of Pharmaceutical Biotechnology, Department of Sports Medicine and Adult Reconstructive Surgery, Nanjing Drum Tower Hospital, The Affiliated Hospital of Nanjing University Medical School, 321 Zhongshan Road, Nanjing 210008 Jiangsu, PR China. ${ }^{3}$ Departments of Laboratory Medicine and Infectious Diseases, Nanjing Drum Tower Hospital, The Affiliated Hospital of Nanjing University Medical School, 321 Zhongshan Road, Nanjing 210008 Jiangsu, PR China. ${ }^{4}$ These authors contributed equally: Wenfang Xiong, Kuo-Yang Sun. ${ }^{凶}$ email: 13770654281@163.com; zgr03summer@126.com; zouxp@nju.edu.cn Edited by Professor Hans-Uwe Simon
} 


\section{MATERIALS AND METHODS \\ Cell culture}

Murine bone marrow-derived macrophages (BMDMs) were prepared as described previously [24]. Mouse femurs and tibias (8-week old, male) were cut at both ends, and then flushed with 1640 medium (Corning) using a syringe. The bone marrow cells filtered through a $70-\mu \mathrm{m}$ strainer were plated on sterile petri dishes and incubated for 7 days in 1640 medium containing $10 \%$ (vol/vol) heat-inactivated fetal bovine serum (FBS) (Biological Industries), penicillin, streptomycin (Gibco), and $20 \mathrm{ng} / \mathrm{ml}$ mouse macrophages colony-stimulating factor (M-CSF) (MCE, HY-P7085). Murine RAW 264.7 macrophages (purchased from the Cell bank of Type Culture Collection of Chinese Academy of Science) were cultured in DMEM medium (WISENT) containing 10\% FBS, penicillin, and streptomycin. Macrophages with a $2-\mathrm{h}$ pretreatment of $2 \mathrm{mM}$ metformin or $20 \mu \mathrm{M} \mathrm{C75}$ were treated with $100 \mathrm{ng} / \mathrm{ml}$ LPS (Sigma, L2630) for $30 \mathrm{~min}$ or $4 \mathrm{~h}$.

\section{Transductions of shRNA}

Two independent shRNA lentiviral constructs were used for knockdown of mouse Fasn. BMDMs $\left(1 \times 10^{6}\right.$ cells per well) or RAW 264.7 macrophages $\left(2 \times 10^{5}\right.$ cells per well) were seeded in six-well plates. Culture medium was replaced with fresh medium containing $10 \%$ FBS on the day before transduction. Cells were transduced with shRNA lentiviral constructs against mouse Fasn or control shRNA constructs according to the manufacturer's instructions.

(the targeting sequence of shFASN-1: CCGGCGTCTATACCACTGCTTACT ACTCGAGTAGTAAGCAGTGGTATAGACGTIITG;

the targeting sequence of shFASN-2: CCGGCCCTTGATGAAGAGGGATC ATCTCGAGATGATCCCTCTTCATCAAGGGTITTG).

\section{Overexpression of Akt (wild type or mutant type)}

The Akt overexpression plasmids of wild type (WT) and mutant type (C60S) were constructed with the assistance of GeneChem Co., Ltd (Shanghai, China) from CV061 vector (CMV-MCS-3FLAG-SV40-Puromycin). Adherent RAW 264.7 macrophages were transfected with either wild or mutant plasmids, using Lipofectamine ${ }^{T M} 3000$ reagent (Invitrogen, L3000015). The processes were as the manufacturer' instructions indicated.

\section{Free fatty acid measurements}

Intracellular free fatty acid content was determined using free fatty acid assay kit (Solarbio, BC0595) as described in the manufacturer's instructions, the principle of which was that free fatty acid could bind with $\mathrm{Cu}^{2+}$ forming copper salt. The copper salt was soluble in chloroform, so it was possible to calculate free fatty acid content when measuring the content of copper solubilized in the organic solvent. Briefly, cell samples were homogenized in lysis buffer provided by the kit. After centrifugation, the supernatant was collected for assaying. The extract was mixed adequately with an organic solvent (n-heptane: methyl alcohol: chloroform $=24: 1: 25$ ) and $\mathrm{CuSO}_{4}$ solution, and then centrifugated to separate the oil phase (upper) from the water phase (lower). The supernatant was pipetted to measure the copper salt, which has bind with free fatty acid. Total protein concentrations were determined using BCA assay (KeyGEN, KGP903) for normalization of free fatty acid content.

\section{Acly-biotin exchange}

Acly-biotin exchange (ABE) assay was based on a previously published protocol [25]. Briefly, samples were homogenized in lysis buffer $(150 \mathrm{mM}$ $\mathrm{NaCl}, 50 \mathrm{mM}$ Tris, $5 \mathrm{mM}$ EDTA, pH 7.4, $1 \mathrm{mM}$ PMSF, protease inhibitor cocktail), which additionally contained $50 \mathrm{mM}$ N-ethylmaleimide (NEM) (Thermo Fisher, 23030). Lysates were sonicated and then incubated overnight at $4{ }^{\circ} \mathrm{C}$ with gentle end-over-end rotation. The supernatants obtained by centrifugation at $12,000 \mathrm{rpm}$ for $20 \mathrm{~min}$ were subject to chloroform-methanol protein precipitation (supernatant: methanol: chloroform: water, 1:4:1.5:3) to remove excess NEM. The precipitate was dissolved in $4 \%$ SDS buffer (4\% SDS, $50 \mathrm{mM}$ Tris, $5 \mathrm{mM}$ EDTA, $\mathrm{pH} 7.4$ ) at room temperature and then divided into two equal portions. One portion was mixed with + HAM buffer ( $\mathrm{pH}$ 7.4); the other portion was added to -HAM buffer ( $\mathrm{pH}$ 7.4) correspondingly. + HAM buffer was a solution including $0.7 \mathrm{M}$ hydroxylamine (Sigma, 438227), $1 \mathrm{mM}$ HPDP-biotin (Thermo Fisher, 21341), 0.2\% Triton X-100, $1 \mathrm{mM}$ PMSF, and protease inhibitor cocktail, whereas -HAM buffer contained $50 \mathrm{mM}$ Tris, $1 \mathrm{mM}$ HPDP-biotin, $0.2 \%$ Triton X-100, 1 mM PMSF and protease inhibitor cocktail. All samples were incubated at room temperature for $1 \mathrm{~h}$ with end-over-end rotation, and then subject to chloroform-methanol protein precipitation again. Protein was solubilized in $2 \%$ SDS buffer and diluted by $1 / 20$, followed by incubation with streptavidin-agarose (Thermo Fisher, 20347) at room temperature for $90 \mathrm{~min}$. The immunoprecipitated beads were washed three times with lysis buffer and eluted with $2 \times$ SDS loading sample buffer.

Immunoprecipitation-ABE (IP-ABE) assay was employed to confirm a palmitoylated protein of interest [26]. The target protein was initially purified by an immunoprecipitation step using an antibody directed against it. The subsequent procedures had a similar principle with ABE assay. Briefly, culture cells were homogenized in lysis buffer containing NEM for $1 \mathrm{~h}$ at $4{ }^{\circ} \mathrm{C}$ and the supernatant was incubated with the primary antibody overnight at $4{ }^{\circ} \mathrm{C}$. On the second day, the protein A/G agarose was added into the supernatant and the incubation lasted another $2 \mathrm{~h}$. The beads were washed three times with lysis buffer $(\mathrm{pH} 7.4)$ and then suspended in hydroxylamine-containing lysis buffer at room temperature for $1 \mathrm{~h}$. The beads were washed three times again and interacted with HPDP-biotin $(4 \mu \mathrm{M}, \mathrm{pH} 6.4)$ at $4{ }^{\circ} \mathrm{C}$ for another hour. The immunoprecipitated samples were analyzed by western blot using anti-Akt antibody and streptavidin-HRP (Beyotime, A0303).

\section{Subcellular fractionation}

Cell membrane protein and cytoplasmic protein extraction reagent (KeyGEN, KGP3100) was utilized following its instructions. In brief, cells were harvested in lysis buffer containing protease inhibitor cocktail and DTT. The lysates were incubated on ice for $1 \mathrm{~min}$ and then vortexed for $30 \mathrm{~s}$, which was repeated five or more times to break up over $90 \%$ cells. After centrifugation at $12,000 \mathrm{rpm}$ for $10 \mathrm{~min}$ at $4{ }^{\circ} \mathrm{C}$, the obtained supernatants were the cytoplasmic protein, whereas the pellets were subsequently homogenized in extraction buffer to get the membrane protein. All protein was mixed with SDS loading buffer and heated at $95^{\circ} \mathrm{C}$ for $10 \mathrm{~min}$.

\section{Western blotting}

Cells or tissues were harvested and homogenized on ice using RIPA lysis buffer (Beyotime, P0013B) containing protease inhibitor cocktail (Roche, 04693132001), phosphatase inhibitor cocktail (Roche, 04906837001) and PMSF (KeyGEN, KGP610). After centrifugation at $12,000 \mathrm{rpm}$ for $20 \mathrm{~min}$ at $4{ }^{\circ} \mathrm{C}$, the supernatant concentrations were determined by BCA assay (KeyGEN, KGP903) according to its instructions. Equal amounts of protein were subjected to $8-12 \%$ SDS-PAGE gels (BIO-RAD, 79-06-1) and then transferred to polyvinylidene difluoride membranes (Millipore, IPVH00010). The membranes were blocked in tris-buffered saline with $0.1 \%$ Tween (TBS-T) containing $5 \%$ non-fat milk or bovine serum albumin for $1 \mathrm{~h}$ at room temperature, and then incubated with primary antibodies overnight at $4{ }^{\circ} \mathrm{C}$ followed by interaction with HRP-linked secondary antibodies on the second day. Specific signaling was generated using chemiluminescent substrate (Share-Bio, sb-wb012) and recorded with a CCD camera (Tanon, 5200 Multi).

\section{Immunofluorescence}

Cells seeded on glass dishes were fixed with $4 \%$ paraformaldehyde (vol/vol in PBS) for $30 \mathrm{~min}$. The fixed cells were washed twice with PBS, and then permeabilized using $0.2 \%$ Triton X-100 (vol/vol in PBS). Next the cells were blocked with $5 \%$ bovine serum albumin (in PBS) for $30 \mathrm{~min}$, followed by incubation with primary antibodies overnight at $4{ }^{\circ} \mathrm{C}$. On the second day, the cells continued to interact with secondary antibodies at room temperature in the dark for $1 \mathrm{~h}$ after removing excess primary antibodies. Immunoreactive signaling was visualized with fluorescence microscope (ZEISS).

\section{RNA isolation and quantitative RT-PCR}

Total RNA was isolated from cultured cells or tissues using Trizol reagent (Takara, 9109). Reverse transcription reactions were performed to synthesize cDNA from $1 \mu \mathrm{g}$ of total RNA using HiScript III Q RT SuperMix (Vazyme, R323-01) as described in the manufacturer's instructions. A $20 \mu \mathrm{l}$ mixture containing diluted CDNA, gene-specific primers and ChamQ Universal SYBR qPCR Master Mix (Vazyme, Q711-02) was subjected to quantitative RT-PCR. The temperature and time were set according to the manufacturer's protocol. The $2-\delta \delta C T$ was calculated to determine the relative mRNA levels of a target gene.

\section{DSS-induced colitis mouse model}

Six-week-old male C57BL/6 mice (purchased from Nanjing Medical University) were housed under specific pathogen-free conditions at 
Nanjing Drum Tower Hospital. Colitis was induced by adding 2.5\% DSS (MP Biomedicals, 02160110) to their drinking water from day 0 to day 8 and 5-10 mice per group were recommended [27]. Mice were randomly divided into five groups, consisting of water group, DSS group, metformin1 group, metformin-2 group, and C75 group. The latter four groups all drunk water containing DSS. $100 \mathrm{mg} / \mathrm{kg}$ metformin hydrochloride (Sigma, 1396309), $200 \mathrm{mg} / \mathrm{kg}$ metformin or $5 \mathrm{mg} / \mathrm{kg}$ C75 (MCE, HY-12364) in $200 \mu \mathrm{l}$ saline was daily administered to metformin-1 group, metformin-2 group, or C75 group by intraperitoneal injection, starting from day 3 . The dose of these drugs was based on previous studies [28, 29]. Animal care and handling was approved by the Institutional Animal Care and Use Committee of the Affiliated Drum Tower Hospital of Nanjing University Medical School (Nanjing, China), and was performed following the guidelines set by the Animal Care Committee.

\section{Assessment of disease activity}

Weight changes and characteristics of feces were monitored daily throughout the experiment [27]. The extents of weight loss, diarrhea, and fecal bleeding were separately quantitively recorded as a $0-4$ score [30]. The scoring criteria were conducted as below. Weight loss: 0 points, $<5 \%$ weight loss; 1 point, $5-10 \%$ weight loss; 2 points, $10-15 \%$ weight loss; 3 points, 15-20\% weight loss; 4 points, $>20 \%$ weight loss. Stool consistency: 0 points, pellets; 2 points, paste-like/semi-formed stool; 4 points, liquid stool. Fecal bleeding: 0 points, no bleeding; 2 points, slightly bleeding; 4 points, gross bleeding. The disease activity index (DAl) was calculated by combining scores of the three signs above. All mice were euthanized on day 8 and the colons were collected for length measurement, histopathological analysis, LPMCs isolation, etc. Assessment of disease activity (including DAl score, length measurement and histopathological analysis) were conducted by two blinded individuals.

\section{Immunohistochemistry}

Paraffin-embedded colonic sections of mice were deparaffinized using xylene, and rehydrated through graded ethanol. Immunohistochemistry kit (MXB Biotechnologies, KIT-9720) was used according to its manufacturer's instructions for next processes. Briefly, $3 \%$ (vol/ $/ \mathrm{vol}) \mathrm{H}_{2} \mathrm{O}_{2}$ in methanol was firstly utilized to block endogenous peroxidase. Then sections immersed in citrate buffer were heated in microwave for $15 \mathrm{~min}$ to retrieve antigen. After cooling to room temperature, sections were blocked using goat serum and then incubated with primary antibodies overnight at $4{ }^{\circ} \mathrm{C}$, followed by biotin-conjugated secondary antibodies incubation and subsequent streptavidin-HRP incubation. Immunoreactive tissues were visualized with DAB (MXB Biotechnologies, DAB-0031) as a chromogen and hematoxylin as counterstain.

\section{Histopathological analysis}

Formalin-fixed and paraffin-embedded colon tissues were sectioned $5 \mu \mathrm{m}$ thick and stained with hematoxylin and eosin (H\&E). The histopathological analysis focused on colonic epithelial damage and inflammatory cells infiltration, quantitively with a total score ranging from 0 to 12 [29]. Colonic epithelial damage was described as below: 0 points, normal; 1 point, hyperproliferation, irregular crypts, and goblet cell loss; 2 points, mild to moderate crypt loss (10-50\%); 3 points, severe crypt loss (50-90\%); 4 points, complete crypt loss but intact surface epithelial; 5 points, small- to medium-sized ulcer ( $<10$ crypt widths); 6 points, large ulcer ( $\geq 10 \mathrm{crypt}$ widths). Inflammatory cell infiltration in mucosa, submucosa, and muscle/ serosa was assessed separately [mucosa ( 0 points, normal; 1 point, mild; 2 points, modest; and 3 points, severe); submucosa ( 0 points, normal; 1 point, mild to modest; and 2 points, severe); muscle/serosa ( 0 points, normal; and 1 point, mild to severe)]. Scores for epithelial damage and infiltration were added together in order to evaluate the severities from the perspective of histopathology.

\section{LPMCs isolation}

Murine LPMCs were isolated according to a previous protocol published in Nature [31]. In brief, the freshly obtained colons were cut into $4-5 \mathrm{~cm}$ long pieces and cleared by flushing colonic cavity with a syringe filled with sterile PBS. Then we opened the colons longitudinally in order to wash them cleaner and exposed the mucosa more thoroughly. Colon pieces were sliced smaller $(1 \mathrm{~cm}$ long) and incubated in pre-digestion solution containing $5 \mathrm{mM}$ EDTA and $1 \mathrm{mM}$ DTT (Roche, 10197777001) to separate epithelial cells. Finally, the remaining pieces were digested with collagenase D (Roche, 11088858001) under slow rotation and passed through a $40-\mu \mathrm{m}$ cell strainer to collect LPMCs. The tissues could be digested several times according to the actual situation. LPMCs could be collected for further experiments.

\section{Antibodies and primers}

Anti- $\beta$-actin (Sigma, A5441), anti-GAPDH (Cell Signaling Technology, 5174), anti-a-tubulin (Cell Signaling Technology, 2144), anti-Na ${ }^{+}-\mathrm{K}^{+}$ATPase (proteintech, 14418-1-AP), anti-ACACA (Cell Signaling Technology, 3662), anti-ACLY (proteintech, 15421-1-AP), anti-FASN (Cell Signaling Technology, 3180), anti-AMPK (Cell Signaling Technology, 2532), anti-p-AMPK (Cell Signaling Technology, 2535), anti-p-ACACA (Cell Signaling Technology, 3661), anti-NF-kB p65 (Cell Signaling Technology, 8242), anti-p-NF-kB p65 (Cell Signaling Technology, 3033), anti-Akt (Cell Signaling Technology, 4691), anti-p-Akt (Cell Signaling Technology, 4060), anti-mTOR (Cell Signaling Technology, 2983), anti-p-mTOR (Cell Signaling Technology, 5536), anti-p38 (Cell Signaling Technology, 8690), anti-p-p38 (Cell Signaling Technology, 4511), anti-ERK (Cell Signaling Technology, 9102), anti-p-ERK (Cell Signaling Technology, 4370), anti-JNK (Cell Signaling Technology, 9252), anti-p-JNK (Cell Signaling Technology, 4306).

Infa (forward GCCTCTTCTCATTCCTGCTT, reverse TGGGAACTTCTCAT CCCTTTG), II-1 $\beta$ (forward TGGCAACTGTTCCTG, reverse GGAAGCAGCCCTTC ATCTT), II-6 (forward GCCTTCTTGGGACTGATGCT, reverse TGCCATTGCAC AACTCTITTC), Nirp3 (forward ACCAGCCAGAGTGGAATGAC, reverse ACCT GCTTCTCACATGTCGT), Cox-2 (forward GCCTACTACAAGTGTTCTITITGCA reverse CATTTGTTTGATTGTTCACACCAT), Nos2 (forward AATCTTGGAGCG AGTTGTGG, reverse CAGGAAGTAGGTGAGGGCTTG), $\beta$-actin (forward GT GACGTTGACATCCGTAAAGA, reverse GCCGGACTCATCGTACTCC).

\section{Statistical analysis}

Statistical analysis was performed using GraphPad Prism 7. All data ware presented as mean \pm SD. The difference between the two groups was analyzed by $t$-test (two-tailed, unpaired), while multiple groups were analyzed by one-way ANOVA (with post hoc comparison using Dunnett's test). Each group represented at least three independent experiments (in vitro) or five animals (in vivo). No samples or animals were excluded from being analyzed. The homogeneity of variance was analyzed by $F$ test. $P<0.05$ were considered statistically significant.

\section{RESULTS \\ The inhibition of FASN downregulates inflammatory responses in macrophages}

To vertify the relationship between de novo fatty acid synthesis and inflammatory responses in macrophages, we established an inflammation model of LPS-triggered macrophages. De novo fatty acid synthesis is regulated by three key lipogenic enzymes, FASN, acetyl-CoA carboxylase (ACACA), and ATP citrate lyase (ACLY) [32], so we firstly detected their protein levels to analyze whether LPS stimulus influenced fatty acid synthesis in macrophages. Indeed, there was an elevation of these mentioned enzymes in macrophages after exposure to LPS (Fig. 1A, B). Furthermore, intracellular free fatty acid also increased (Fig. 1C). As FASN converting acetylCoA and malonyl-CoA into fatty acid (mainly palmitic acid) works in the terminal catalytic step [32], we focused on it and evaluated its influence on macrophage activation through using a FASN inhibitor C75 or shRNA lentiviral constructs against Fasn. C75 treatment downregulated LPS-induced p65 phosphorylation (Fig. 2A, B) as well as the enhanced Tnfa, II-1 $\beta$, and II-6 levels (Fig. 2C, Fig. S1-A). These molecules are typical proinflammatory transcriptional factor and cytokines [33, 34]. In addition, we transduced macrophages with shRNA lentiviral constructs to rule out the off-target influence of C75 (Fig. 2D). Knockdown of FASN got a similar result to $\mathrm{C} 75$ utilization (Fig. 2E-G). Taken together, these results suggested that proinflammatory activation of macrophages depended on enhanced fatty acid synthesis.

\section{Metformin alleviates inflammation through inhibiting FASN/ Akt/p65 pathway in macrophages}

Since metformin has been reported to suppress fatty acid accumulation in liver cells [35], we predicted metformin had a 
A

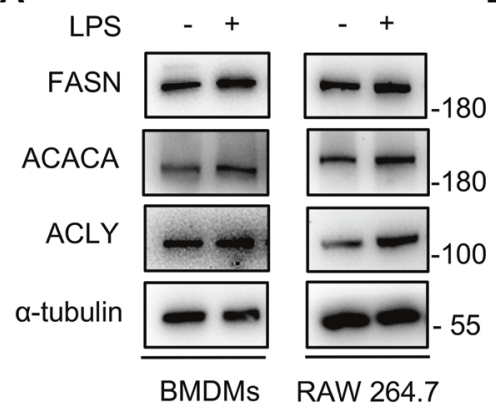

C

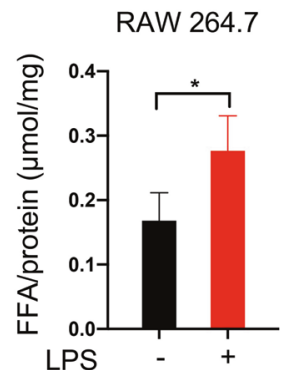

B

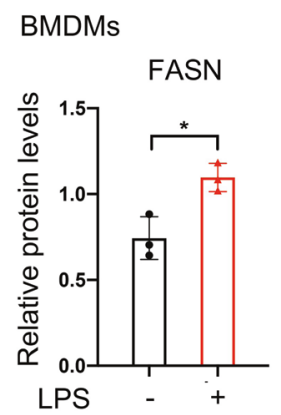

RAW 264.7

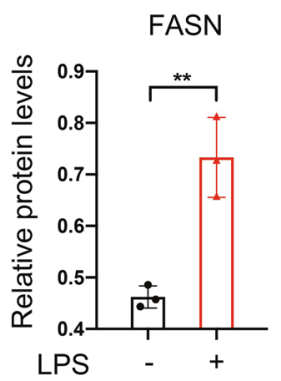

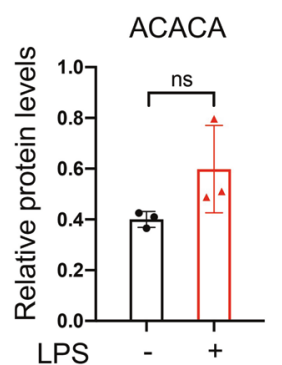

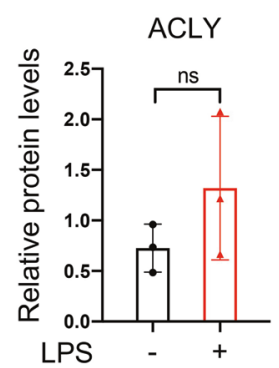

Fig. 1 LPS stimuli promoted de novo fatty acid synthesis pathway in macrophages. A, B Murine bone marrow-derived macrophages (BMDMs) or murine RAW 264.7 macrophages were cultured in the absence or presence of $100 \mathrm{ng} / \mathrm{ml}$ lipopolysaccharide (LPS). The protein levels of fatty acid synthase (FASN), acetyl-CoA carboxylase (ACACA), and ATP citrate lyase (ACLY) were determined by Western blotting. Representative images of western blotting (A) and a statistical analysis of relative protein levels (B). C The intracellular free fatty acid (FFA) was determined by free fatty acid assay kit after exposure to LPS $(n=4)$. Data were all represented as means \pm SD. ${ }^{*} p<0.05,{ }^{* *} p<0.01,{ }^{* * *} p<0.001$, $* * * * p<0.0001$, $t$-test.

similar effect on fatty acid synthesis in macrophages. Indeed, we observed the expression of FASN, ACACA, and ACLY was lower in metformin-treated proinflammatory macrophages than untreated ones (Fig. 3A, B). In addition, metformin treatment, similar to C75, decreased intracellular free fatty acid content in macrophages as well (Fig. 3C). Next, we continued to identify the influence of metformin on macrophage proinflammatory responses. It was found that metformin inhibited LPS-induced enhancement of proinflammatory cytokines (Fig. 3D, Fig. S1-B). The inflammationassociated protein levels of iNOS, COX2, p-p65, and p-IKB also followed a downward trend when macrophages being exposed to metformin (Fig. 3E-H).

Indeed, many articles have claimed that metformin suppresses proinflammatory responses of macrophage through activating AMPK [21]. Therefore, we preliminarily detected the phosphorylation levels of AMPK in macrophages. Consistently, our results also showed that AMPK phosphorylation in macrophages exposed to metformin was enhanced (Fig. S1-C). As our aim was to explain the anti-inflammatory mechanism of metformin from the immunometabolism perspective, we explored other possible signaling pathways instead of the classical one (AMPK).

Combined with the previous data (Figs. 1-3), it was indicated that metformin might alleviate inflammation through suppressing intracellular fatty acid synthesis in macrophages. We further assessed the downstream mechanisms of metformin, and in other words, the downstream pathways affected by fatty acid synthesis. Akt, as a kinase involved in a series of signaling pathways, influences various cell functions and fates, exactly including metabolism and inflammatory responses. In addition, the Akt/ mTOR pathway exactly had a cross talk with AMPK [36]. Therefore, we analyzed the Akt/mTOR pathway in macrophages. Indeed, phosphorylation of Akt (Ser 473) and mTOR was elevated in LPSstimulated macrophages; however, both of them were downregulated under the influence of metformin (Fig. 4A, B; Fig. S1-D).
To further investigate whether metformin-induced inhibition of p-Akt was related to its anti-inflammatory role in macrophages, we chose a PI3K inhibitor, wortmannin, to inhibit the phosphorylation of Akt and observed the following changes of macrophage activation. p65 was less phosphorylated in macrophages treated with wortmannin (Fig. 4C, D), which suggested that Akt phosphorylation played a crucial role in mediating macrophage activation. Based on the previous reports that Akt activation could be modulated by FASN in cancer cells [37, 38], we were curious whether FASN was as well responsible for the phosphorylation of Akt in macrophages. With pharmacological inhibition (C75) or knockdown of FASN (Fasn shRNA lentivirus), LPS-induced p-Akt/pmTOR pathway was significantly downregulated in macrophages (Fig. 4E-H). Consequently, metformin exerted anti-inflammatory effect on macrophages through inhibiting FASN/Akt pathway.

\section{Metformin inhibited Akt activation through suppressing FASN-dependent palmitoylation and its mediated membrane recruitment}

Activated PI3K converts PIP2 to PIP3, which recruits Akt to the plasma membrane [39]. It is precisely on the plasma membrane where two protein kinases, mTORC2 and PDK1, phosphorylate Akt. Membrane attachment is indispensable for the activation of Akt. Traditionally, pleckstrin homology (PH) domain of Akt is responsible for binding to PIP3 [40,41]. The results above showed that metformin and FASN inhibition suppressed Akt phosphorylation. In this part, we continued to uncover that Akt membrane localization was actually disturbed by metformin as well as FASN inhibition, the same as the reduction of Akt phosphorylation (Fig. $5 A-C)$. Palmitic acid can be converted to palmitoyl-CoA and covalently attached to cysteine residues of peptide chains via thioester linkage, which is called S-palmitoylation. Palmitoylation is believed to facilitate membrane localization of protein due to the hydrophobicity of the palmitoyl group $[13,14]$. It is unclear 
A

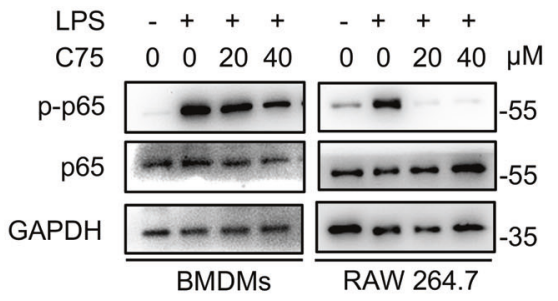

C

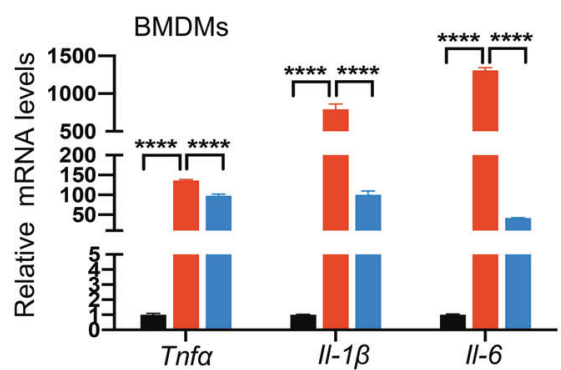

D
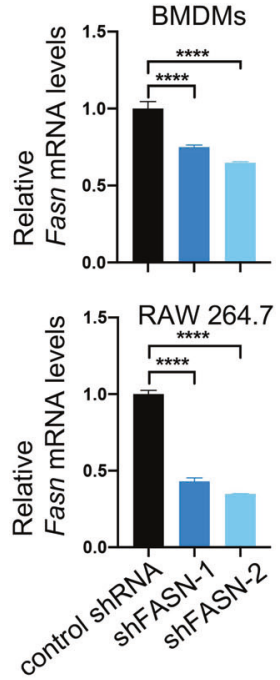

B

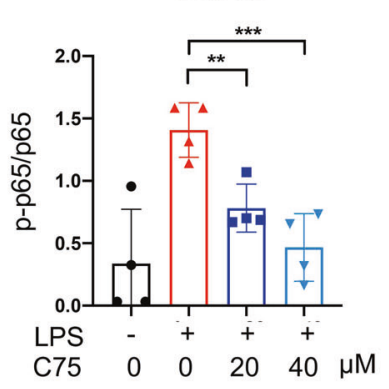

RAW 264.7

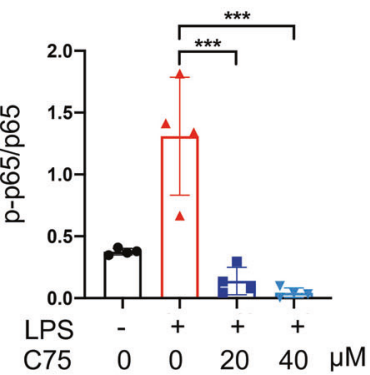

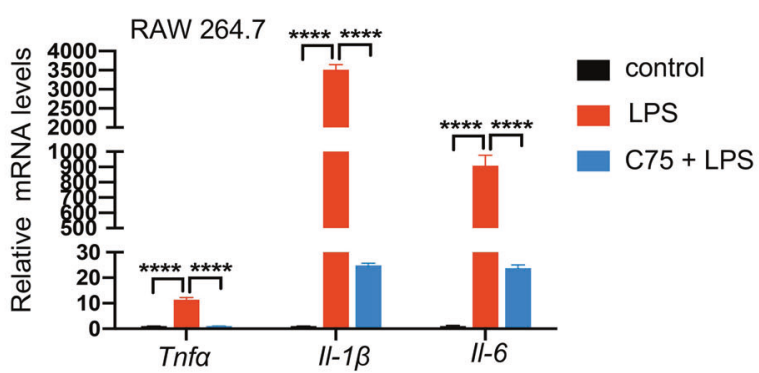

E

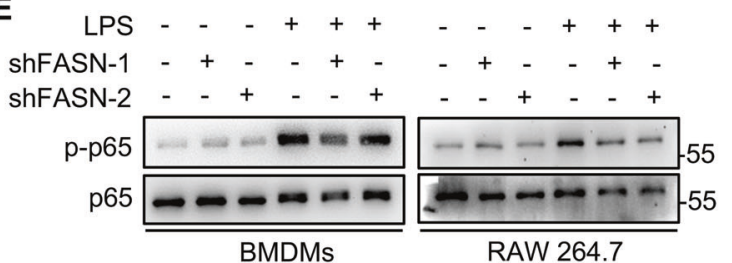

F

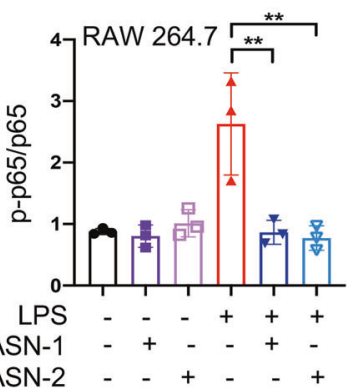

G
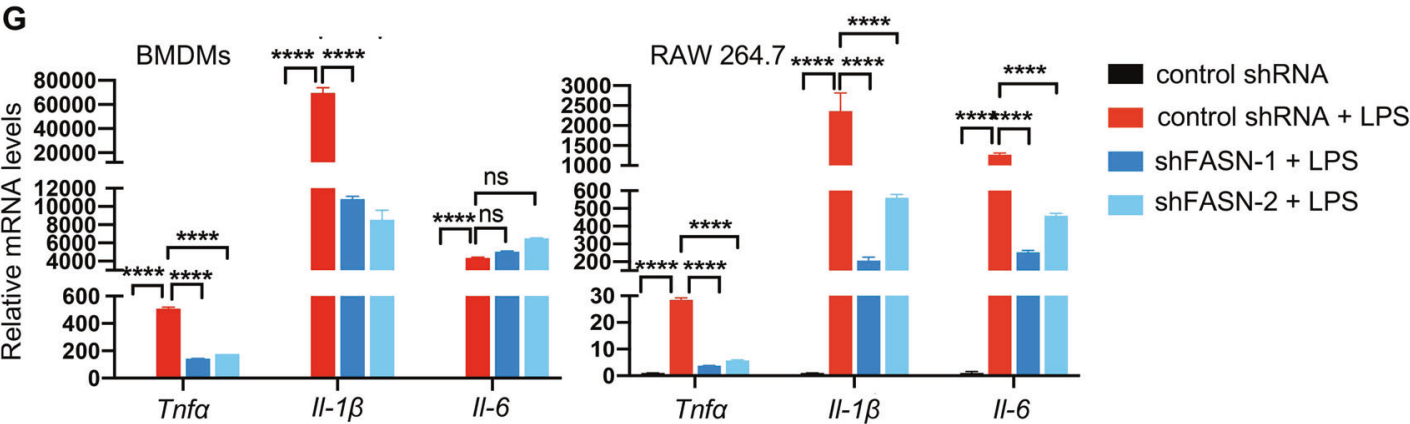

Fig. 2 The inhibition of fatty acid synthesis suppressed proinflammation responses of macrophages. A, B Treatment with various concentrations of C75 (FASN inhibitor) inhibited the phosphorylation of p65 in macrophages exposed to $100 \mathrm{ng} / \mathrm{ml}$ LPS for $30 \mathrm{~min}$, as determined by Western blotting. Representative images of western blotting (A) and quantification of p-p65/p65 ratios (B). C The relative mRNA levels of Tnfa, II-1 $\beta$, and II- 6 were detected by qRT-PCR after treatment with $100 \mathrm{ng} / \mathrm{ml}$ LPS in the presence or absence of $20 \mu \mathrm{M}$ C75 for $4 \mathrm{~h}(n=3)$. D Macrophages were transduced with Fasn shRNA or control shRNA lentivirus constructs. The efficiency of knockdown was confirmed by qRT-PCR ( $n=3$, left) and Western blotting (right). E, F Knockdown of Fasn suppressed p65 phosphorylation in macrophages triggered by $100 \mathrm{ng} / \mathrm{ml}$ LPS for $30 \mathrm{~min}$, as determined by Western blotting. Representative images of western blotting (E) and quantification of p-p65/p65 ratios (F). G Fasn-knockdown macrophages were treated with $100 \mathrm{ng} / \mathrm{ml}$ LPS for $4 \mathrm{~h}$. The mRNA levels of Tnfa, II- $1 \beta$, and II-6 were analyzed by qRT-PCR $(n=3)$. Data were represented as means \pm SD. ${ }^{*} p<0.05,{ }^{* *} p<0.01,{ }^{* * *} p<0.001,{ }^{* * *} p<0.0001$, ANOVA. 
A

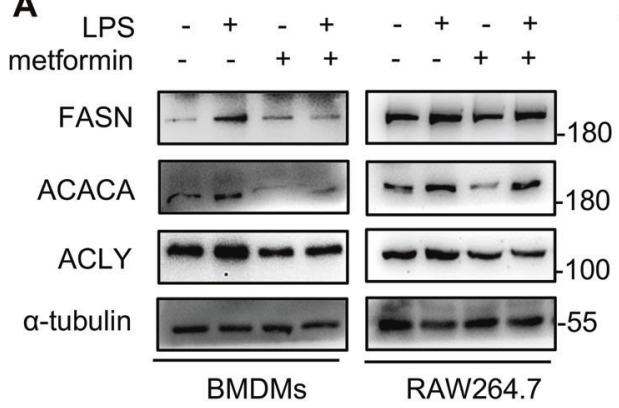

B

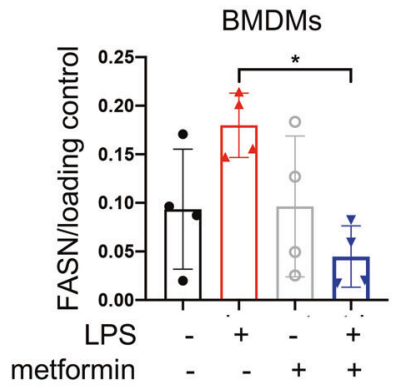

RAW 264.7

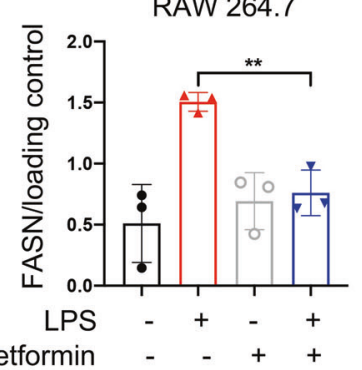

C

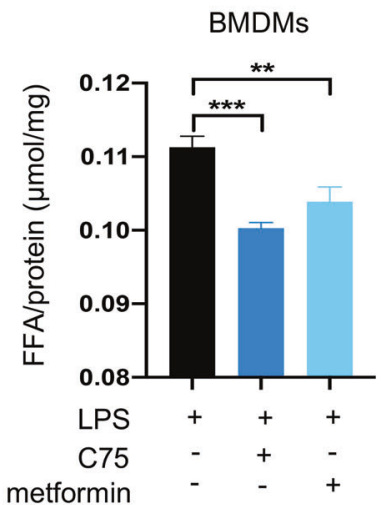

D

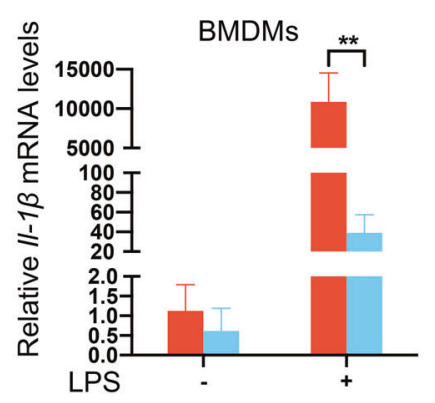

E

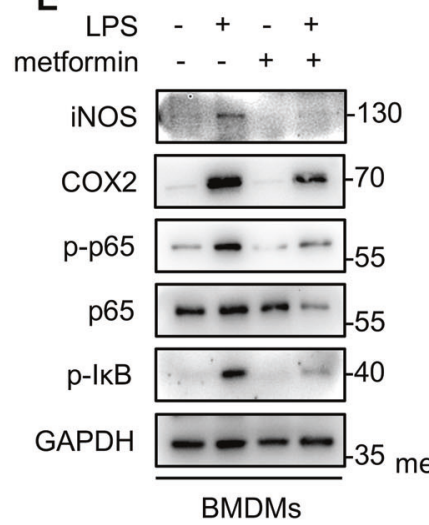

F

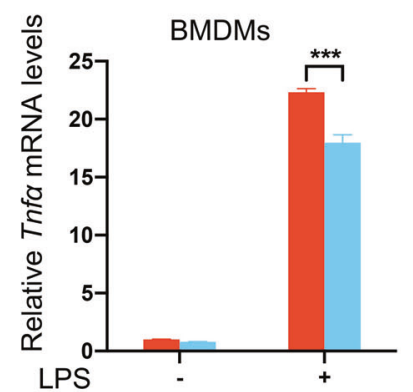

RAW 264.7

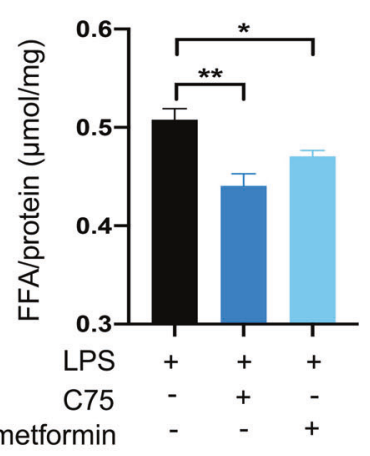

RAW 264.7

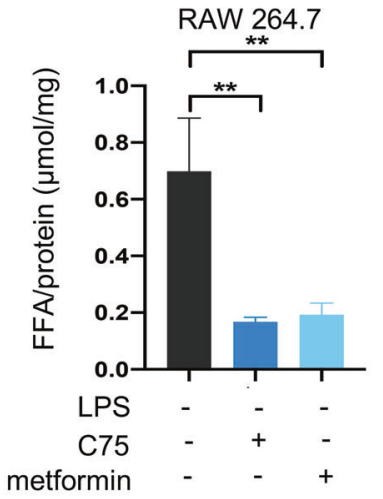


whether palmitoylation facilitates Akt attaching to membranes or not. The software GPS-Palm is able to calculate whether a protein could be palmitoylated and the possible modified amino acid cites [42]. With GPS-Palm, we predicted that Akt was likely to undergo palmitoylation (Fig. S2). Consistently, no matter the endogenous Akt or exogenous Akt overexpressed by flag-Akt plasmids, Akt palmitoylation was both detected according to a classical method
(ABE assay) (Fig. 5D, E). Due to what had been discovered above, we hypothesized that the suppression of FASN caused by metformin contributed to reducing Akt palmitoylation, which further destroyed membrane recruitment and activation (phosphorylation) of Akt. Macrophages treated with C75 or Fasn shRNA lentivirus were regarded as a positive control group. The ABE assay showed that metformin indeed cut down endogenous Akt$$
\text { A }
$$

A LPS - + - + - + +

metformin - $-++-{ }_{-}+$

p-Akt

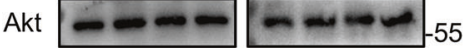

p-mTOR ----180

mTOR

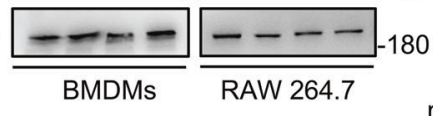

C LPS - + + - + + D

B

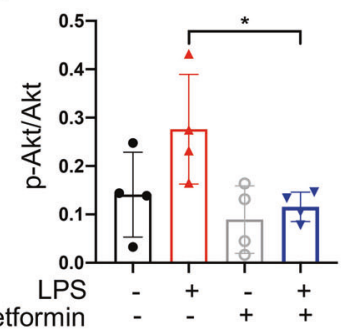

D

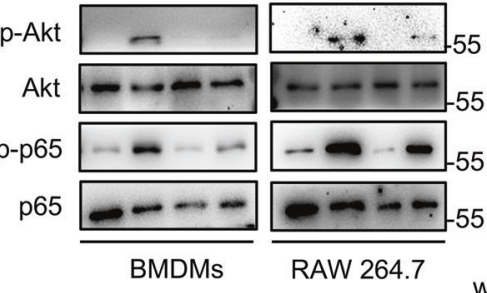

BMDMs

E

$\begin{array}{llllllllll}\text { LPS } & - & + & + & + & - & + & + & + \\ \text { C75 } & 0 & 0 & 20 & 40 & 0 & 0 & 20 & 40 & \mu \mathrm{M}\end{array}$

p-Akt

Akt $=-0-2-5$

$\mathrm{p}$-mTOR $=---180$

mTOR - - - - - - 180

BMDMs RAW 264.7

$\mathbf{F}$

BMDMs
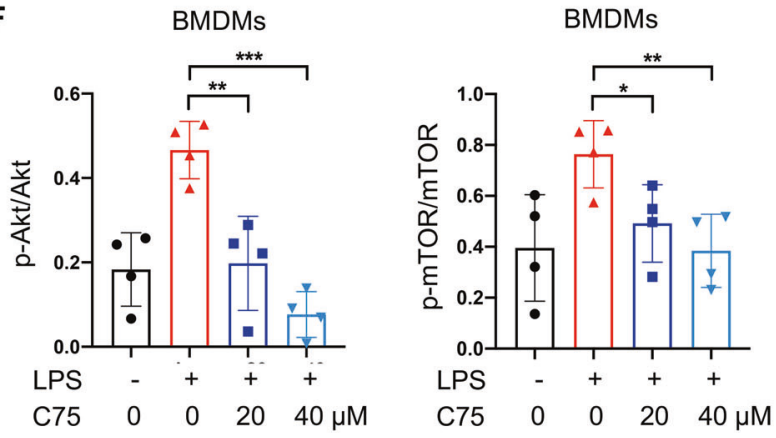

RAW 264.7
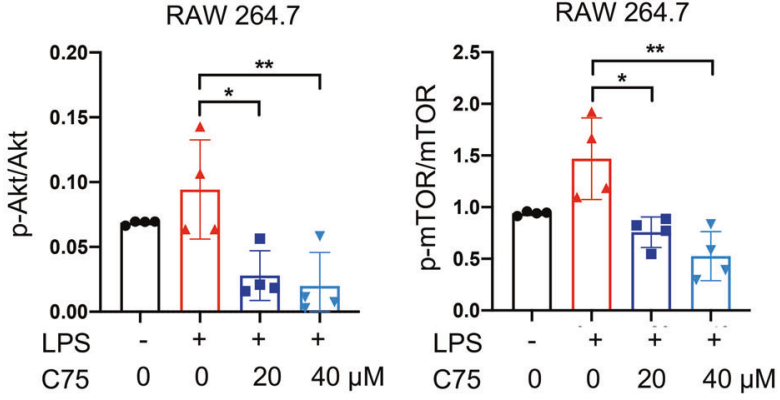

H
G

ShFASN-1

ShFASN-2 - $-+{ }_{-}+$

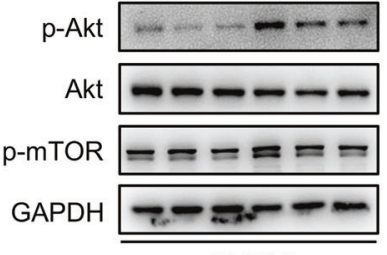

BMDMs

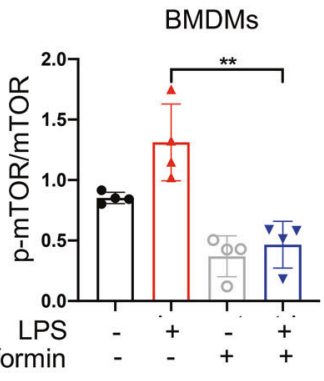

RAW 264.7
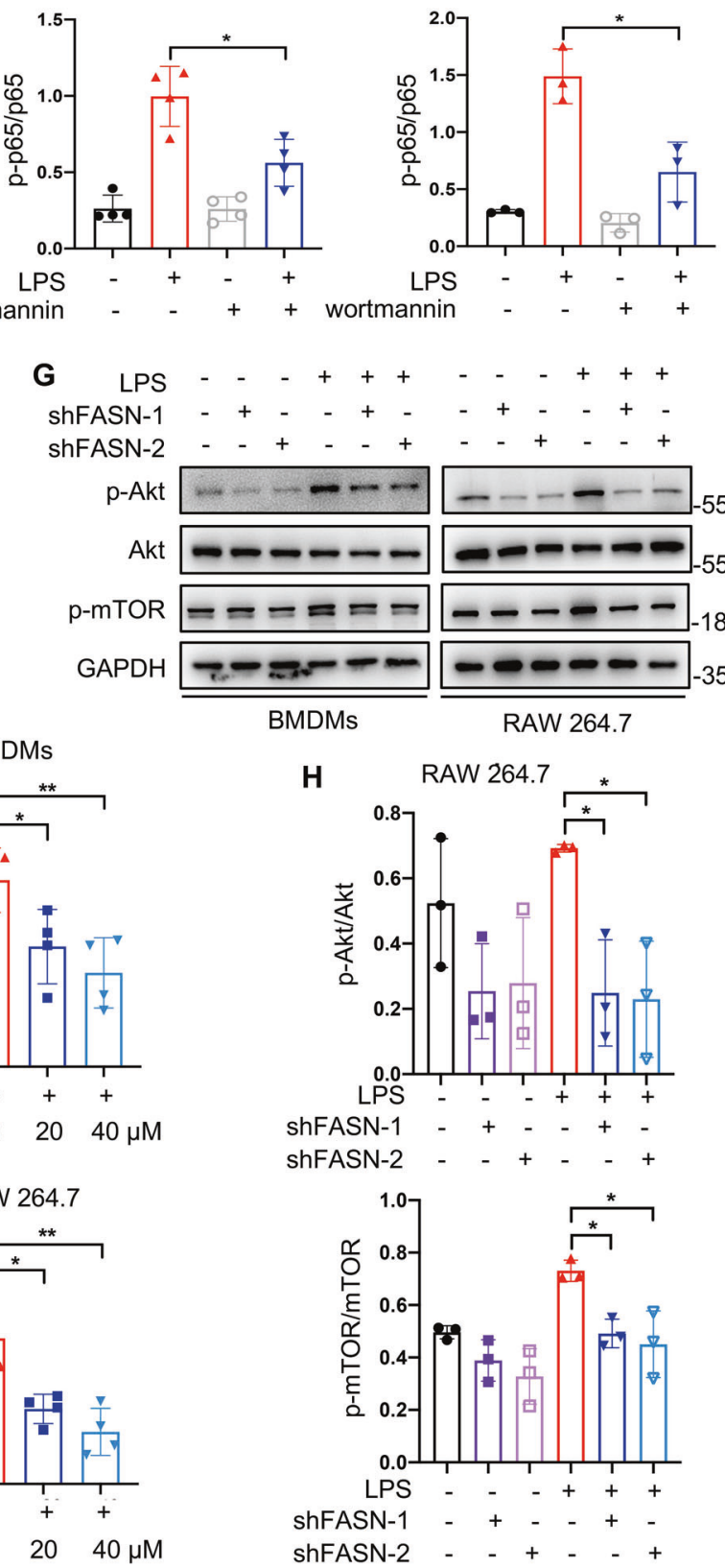
Fig. 4 Metformin alleviated inflammation through inhibiting FASN/Akt/p65 pathway in macrophages. A, B The activation of $p$-Akt/pmTOR were determined by western blotting after macrophages were stimulated with $100 \mathrm{ng} / \mathrm{ml}$ LPS or LPS plus $2 \mathrm{mM}$ metformin. Representative images of western blotting (A) and a statistical analysis of p-Akt/Akt and p-mTOR/mTOR ratios (B). C, D When $20 \mathrm{nM}$ wortmannin (an inhibitor of PI3K/Akt pathway) was used to hinder the activation of Akt, LPS-induced p65 phosphorylation was reduced in macrophages as determined by Western blotting. Representative images of western blotting (C) and a statistical analysis of p-p65/p65 ratios (D). E, F Macrophages were treated with different concentrations of C75 and then Akt phosphorylation was analyzed. Representative images of western blotting (E) and quantification of $\mathrm{p}$-Akt/Akt and p-mTOR/mTOR ratios (F). G, H Macrophages transduced with Fasn shRNA lentivirus were triggered by $100 \mathrm{ng} / \mathrm{ml}$ LPS for $30 \mathrm{~min}$. Akt phosphorylation was analyzed to confirm the regulative effect of FASN on Akt. Representative images of western blotting $(\mathbf{G})$ and quantification of $\mathrm{p}$-Akt/Akt and $\mathrm{p}-\mathrm{mTOR} / \mathrm{mTOR}$ ratios $(\mathbf{H})$. Data were all represented as means $\pm \mathrm{SD}$. ${ }^{*} p<$ $0.05,{ }^{* *} p<0.01,{ }^{* * *} p<0.001,{ }^{* * *} p<0.0001$, ANOVA or $t$-test.

palmitoylation, which was validated again by IP-ABE assay (Fig. $5 \mathrm{~F}-\mathrm{H})$.

To further investigate the role of palmitoylation in Akt activation, 2-bromopalmitate (2-BP) (Sigma, 21604), an inhibitor of palmitoylation, was utilized to decrease Akt palmitoylation. As we speculated, inhibiting palmitoylation suppressed the membrane distribution of Akt, as well as the phosphorylation of Akt, p65, and IKB in macrophages (Fig. 6A-C). These results indicated that Akt palmitoylation promoted it to be recruited to membrane, and further be phosphorylated. In addition to the possibility of Akt palmitoylation, the software GPS-Palm (Fig. S2) also predicted the probably modified cysteines of Akt, among which C60 (the sixtieth cysteine) got the highest score. To verify the prediction above and further confirm the importance of Akt palmitoylation to its activation, we constructed two types of flag-Akt plasmids, wild type and mutant type (C60S). As Fig. 6D, E exhibited, Akt was less palmitoylated when the sixtieth cysteine (C60) was mutated, which not only verified $\mathrm{C} 60$ was one of the palmitoylated sites, but also implied there existed other cysteines able to be palmitoylated as C60 mutant Akt still had a low level of palmitoylation. Apart from the palmitoylation level, the phosphorylation and membrane attachment of $\mathrm{C} 60$ mutant Akt were as well inhibited, compared with the wild type (Fig. $6 \mathrm{~F}-\mathrm{H}$ ). These data complemented the above indication that Akt palmitoylation promoted it to be recruited to membrane and further be phosphorylated. Taken together, metformin could inhibit Akt activation through suppressing FASN-dependent palmitoylation and its mediated membrane recruitment.

\section{MAPK pathways are downstream signaling of FASN and Akt}

To further understand the anti-inflammatory mechanism of metformin, we additionally analyzed the MAPK pathways in macrophages (Fig. S3), which have been identified involved in proinflammatory responses [43]. We firstly validated that metformin significantly suppressed LPS-induced phosphorylation of ERK and JNK (Fig. S3-A). Next, in order to reveal the functional effect of FASN on the regulation of MAPK pathways, we investigated whether knockdown of FASN by Fasn shRNA transduction could reduce the activation of MAPK pathways. The LPS-stimulated phosphorylation of MAPK was indeed downregulated in macrophages transduced with Fasn shRNA, in comparation with control shRNA transduced cells (Fig. S3-B). Similarly, the inhibition of FASN activity by $\mathrm{C} 75$ decreased the phosphorylation of p38, ERK, and JNK (Fig. S3-C). Lastly, we wondered whether Akt, as a downstream molecule of FASN, acted on the upstream of MAPK pathways. While using wortmannin to inhibit Akt activation, we observed that inhibition of Akt activation suppressed the phosphorylation of ERK and JNK in LPS-stimulated macrophages (Fig. S3-D). Consequently, it was suggested that MAPK pathways were downstream signaling of FASN/Akt and inhibited by metformin under inflammatory conditions.

\section{Metformin and C75 ameliorates DSS-induced colitis in mice} Inflammatory bowel disease (IBD) is a chronic, incurable disease of the digestive tract, including Crohn's disease and Ulcerative colitis. An increasing number of studies have found that an abundance of proinflammatory macrophages in inflammatory sites contributes to IBD pathogenesis. Therefore, we evaluated the therapeutic effect of metformin on colitis. As DSS-induced colitis is a chemical damage associated inflammatory process, where adaptive immune is dispensable to colitis progression, this model is suitable for studying innate immune mechanisms and the influence of metformin on innate immune cells in vivo [44]. Weight loss, DAl, and shortening of colon length are general indicators to reflect the severity of DSS-induced colitis [27]. Animal experiment was conducted as Fig. 7A. Colitis mice given metformin or C75 showed significantly diminished weight loss as compared with non-treated colitis mice (Fig. 7B). Consistently, the colon from colitis mice was shorter with a smaller cecum and less stool retained inside comparing to the one from the noncolitis mice (Fig. 7C, D). However, metformin or C75 treatment recovered the colon to their normal condition, nearly the same to the non-colitis one (Fig. 7C, D). We next calculated the DAl scores. The treated groups obtained a lower DAI score than non-treated group (Fig. 7E). In order to analyze the colonic inflammation accurately, histopathological evaluation was conducted on the proximal portions of colons. Large ulceration and massive infiltration of inflammatory cells were evident in colon tissues exposed to DSS (Fig. 7F, G). Conversely, colitis colons with metformin or C75 had markedly attenuated colonic epithelial damage and inflammatory infiltration, indicating their effectiveness on more than clinical parameters (Fig. 7F, G). The quantitative scoring of the histological analysis showed that the mice administrated with $200 \mathrm{mg} / \mathrm{kg}$ metformin got a significantly lower score than the colitis mice (Fig. 7F). Therefore, these improvements directly showed that metformin or inhibition of FASN ameliorated macrophages-involved colitis.

\section{Metformin suppresses proinflammatory responses of colonic LPMCs in DSS-induced colitis mice}

We isolated LPMCs from the total colon to demonstrate whether the metformin-induced attenuation of colonic inflammation was, at least partly, attributed to its effective inhibition on colonic macrophages. Similar to in vitro experiments, intracellular free fatty acid of LPMCs seemed to be downregulated by metformin treatment, though without statistical significance (Fig. S4). Since Tnfa, II-1 $\beta, 11-6$, Cox-2, and Nos 2 were crucial proinflammatory molecules involved in the pathogenesis of IBD, we measured their expressions of LPMCs. Their mRNA levels significantly increased after DSS exposure; but on the contrary, the administration of metformin or $\mathrm{C} 75$ decreased the elevation (Fig. 8A). Furthermore, metformin restrained activation of the IKB/p65 pathway in LPMCs (Fig. 8B). The elevated protein levels of COX2 and iNOS in LPMCs of colitis mice were disrupted when receiving metformin administration (Fig. 8B). In line with the above results derived from LPMCs, IHC of colon slices exhibited that colitis mice receiving metformin had lower levels of TNFa in colons (Fig. 8C). To further evaluate the changes of Akt activation and palmitoylation under the influence of metformin in vivo, we detected both levels in LPMCs (Fig. 8D-G). In consistence with in vitro results, metformin utilization reduced Akt phosphorylation (Fig. 8D, E) as well as Akt palmitoylation (Fig. 8F, G). 
A
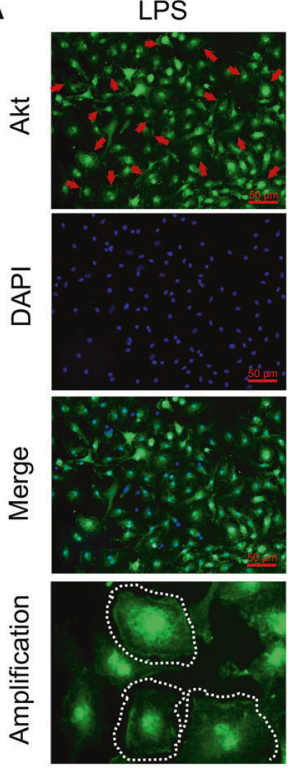

B

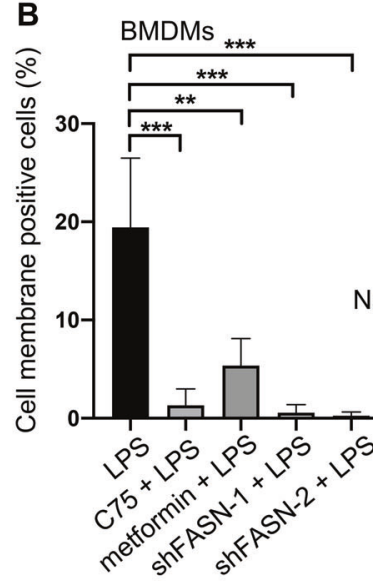

D

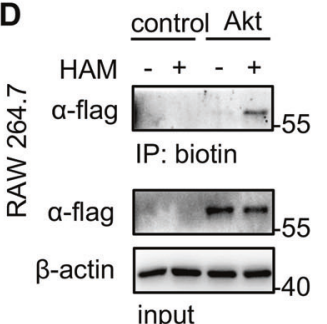

C75 + LPS
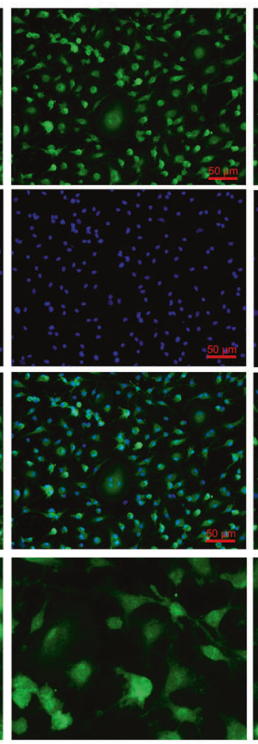

C

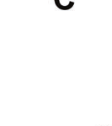

metfor
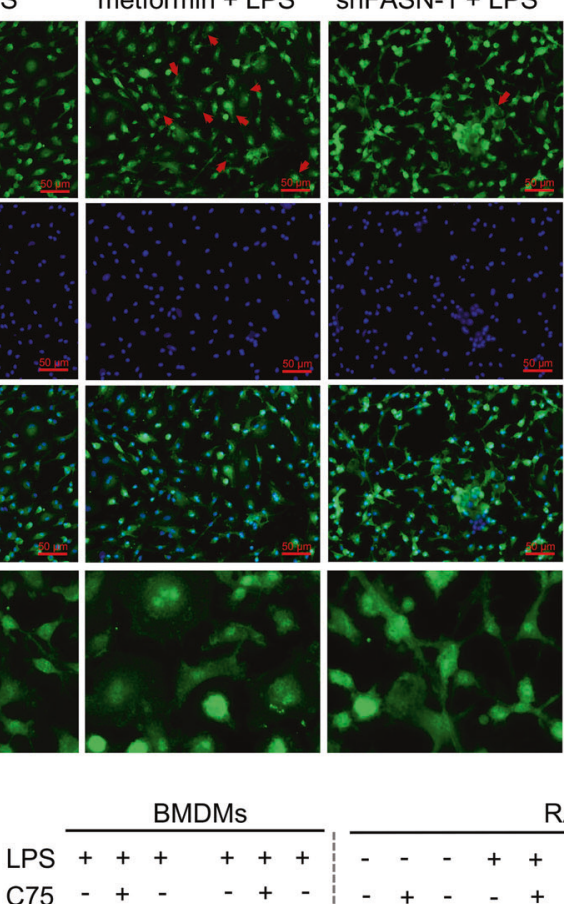

shFASN-2 + LPS
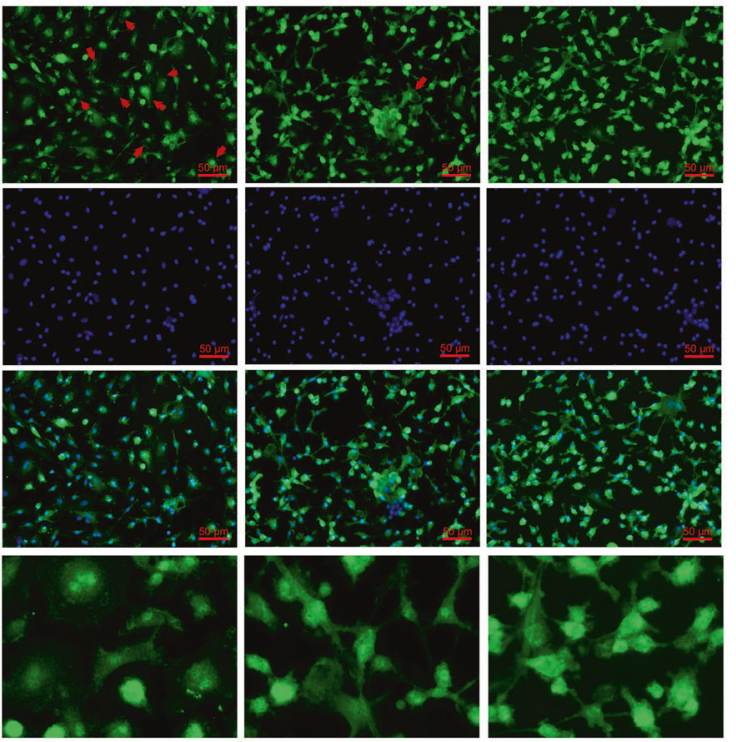

RAW 264.7

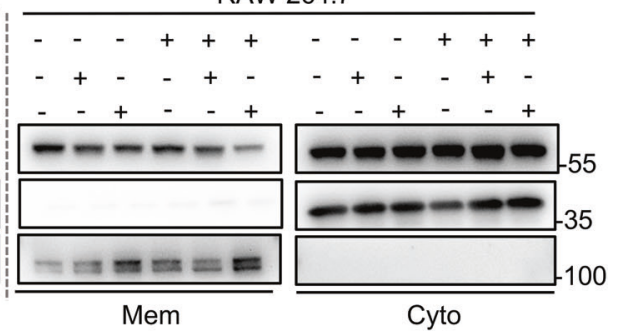

GAPDH

$\mathrm{Na}^{+}, \mathrm{K}^{+}$-ATPase

Akt $=-$
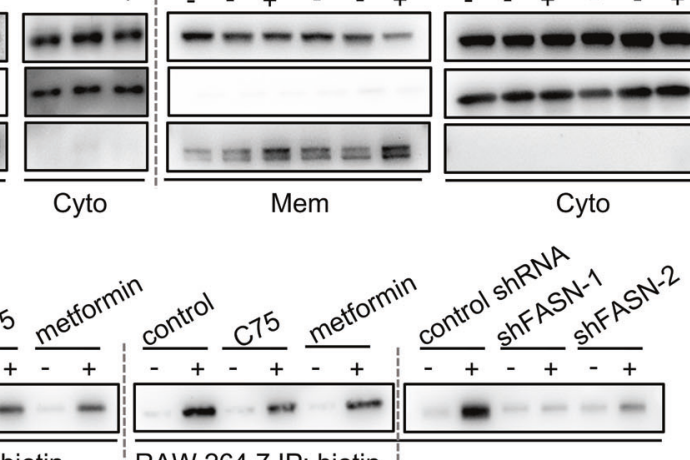

F

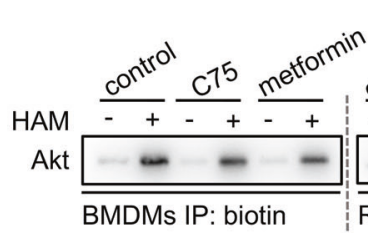

RAW 264.7 IP: biotin
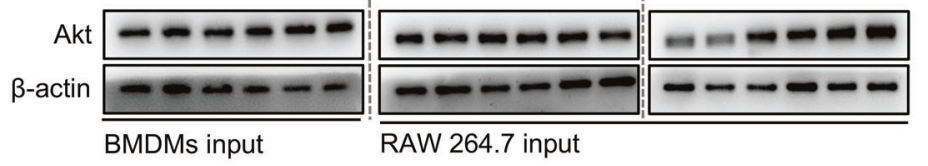

G HAM $\frac{\text { control }^{-}+\frac{\mathrm{C}^{15}}{-}+\frac{\text { metormin }}{-+}}{-+}$
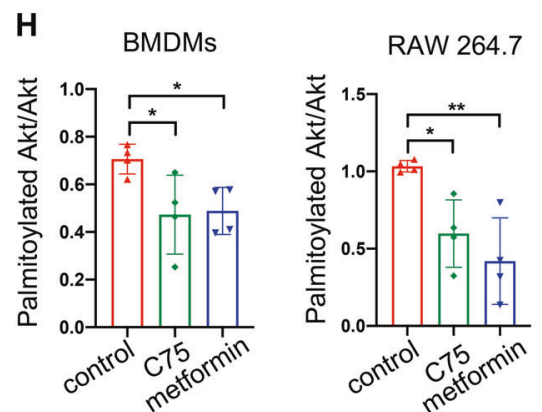

Fig. 5 Metformin and FASN regulated the membrane attachment of Akt and its palmitoylation. Macrophages in the presence of LPS were treated with metformin, C75 or Fasn shRNA lentivirus. A-C The membrane localization of Akt in response to a 15-min LPS treatment was determined by immunofluorescence (A-B) and Western blotting (C). D, E Akt possessed a posttranslational modification called palmitoylation, as determined in RAW 264.7 by acly-biotin exchange (ABE) method. The palmitoylation of exogenously-overexpressed Akt (D) and endogenous Akt (E). F-H The effect of metformin and FASN inhibition on Akt palmitoylation was determined by ABE method (F) and then confirmed again by immunoprecipitation (IP)-ABE method (G). The ratio of palmitoylated Akt/Akt was quantified (H). Data were represented as means \pm SD. ${ }^{*} p<0.05,{ }^{* *} p<0.01,{ }^{* * *} p<0.001,{ }^{* * * *} p<0.0001$, ANOVA. 
A

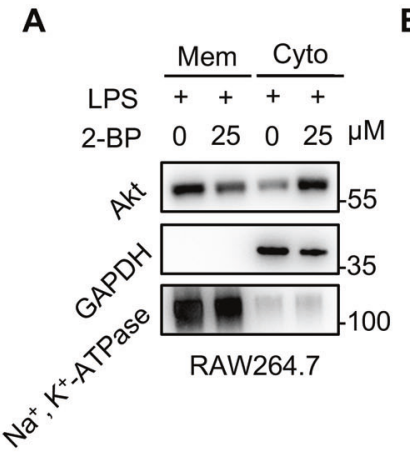

D

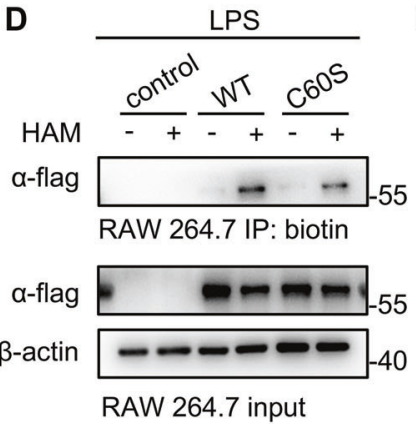

B

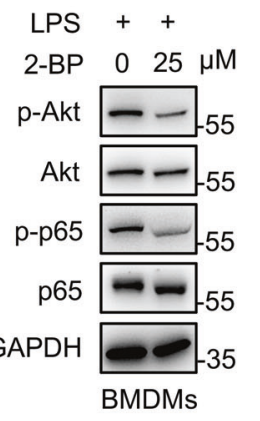

E

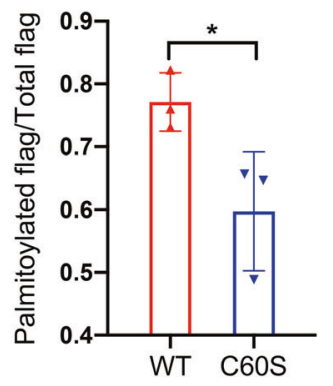

Akt
LPS - - - + + +

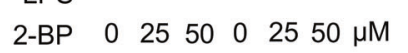
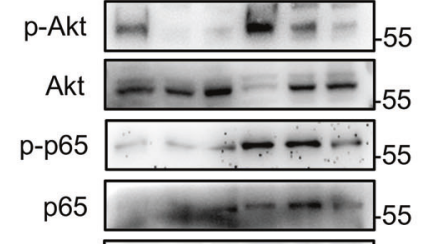

$\mathrm{p}-\mathrm{K} \mathrm{B}$

$\mathrm{IKB}=-0$

$\beta$-actin - -

RAW264.7

F

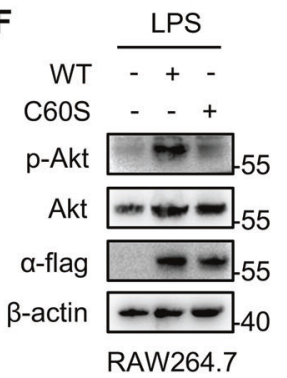

C

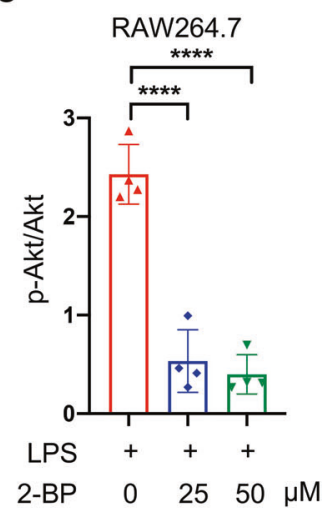

G

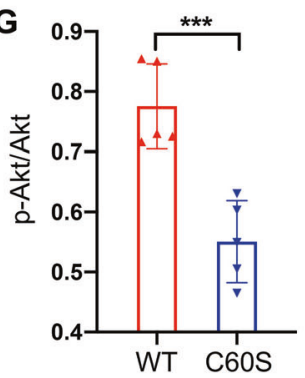

H

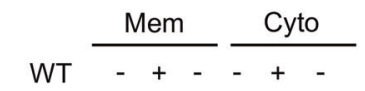

C60S - + + - +

$\alpha^{20}--55$

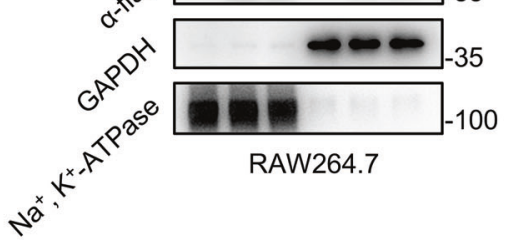

Fig. 6 The palmitoylation of Akt promoted its membrane distribution and phosphorylation (activation). A 2-bromopalmitate (2-BP, an inhibitor of palmitoylation) reduced the membrane localization of Akt in macrophages. B, C The activation of Akt was as well suppressed by 2-BP in macrophages. Representative images of western blotting (B) and quantification of a p-Akt/Akt ratio (C). D, E RAW 264.7 macrophages were transfected with flag-Akt plasmids to detect the palmitoylated site of Akt. The palmitoylated levels of mutant Akt (C60S) were lower than those of the wild type. Representative images of western blotting (D) and quantitative ratios of palmitoylated flag/total flag (E). F, G The mutant Akt (C60S) was less phosphorylated than the wild type. Representative images of western blotting (F) and quantitative ratios of p-Akt/ Akt (G). H The mutant Akt (C60S) was less recruited to membrane than the wild type. Data were represented as means \pm SD. ${ }^{*} p<0.05,{ }^{* *} p<$ $0.01,{ }^{* * *} p<0.001,{ }^{* * * *} p<0.0001$, ANOVA or $t$-test.

\section{DISCUSSION}

Numerous studies on the relationship between fatty acid and inflammation have focused on obesity associated diseases, such as atherosclerosis, diabetes, and non-alcoholic fatty liver disease. They have revealed that immune cells can be activated by exogenous fatty acid derived from blood, adipocytes or hepatocytes $[7,8]$. However, the contribution of endogenously synthesized fatty acid in immune cells to inflammatory responses remains a limited understanding. Most adult tissues and cells express low levels of FASN and generally obtain free fatty acid from the diet uptake [45]. Nonetheless, higher levels of FASN have been detected in various types of cancer and demonstrated a correlation with malignant degree, which implies probable dysregulation of FASN in some pathological conditions [46-49].
As immunometabolism has been extensively investigated in immune cells, emerging evidence suggests that FASN-generated endogenous fatty acid is an important regulator of immune responses and immune cell fate $[2,6]$. T helper 17 (TH17) cells depend on endogenous fatty acid synthesis to produce phospholipids for cellular membranes [50, 51]. FASN-mediated de novo fatty acid synthesis affects the retention of plasma membrane cholesterol and Rho GTPase trafficking, which is important for adhesion, migration and activation of macrophages in diabetes [10]. Fatty acid synthesis is also required for macrophage colonystimulating factor (M-CSF)-induced differentiation of human monocytes [9]. The mitochondrial uncoupling protein-2 (UCP2) promotes NLRP3 inflammasome activation through upregulating FASN and its mediated fatty acid synthesis [52]. In line with these discoveries, we observed that enhanced expression of FASN was 
A

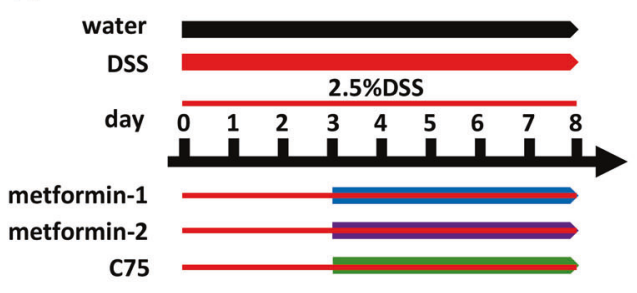

C
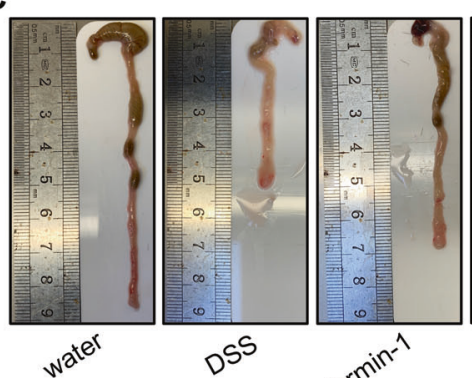<smiles>C1C[C@H]2CC[C@H](C1)C2</smiles>

E

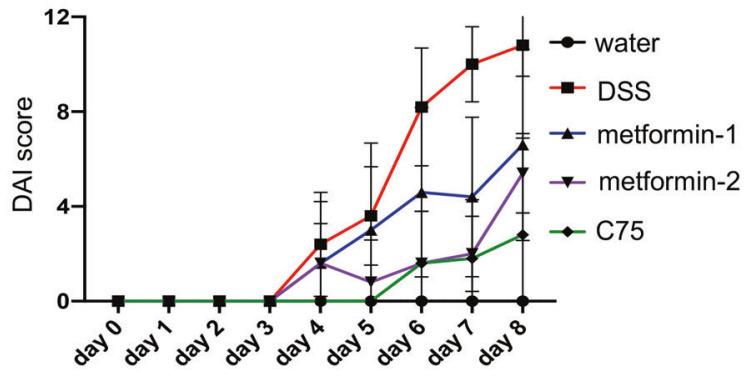

G
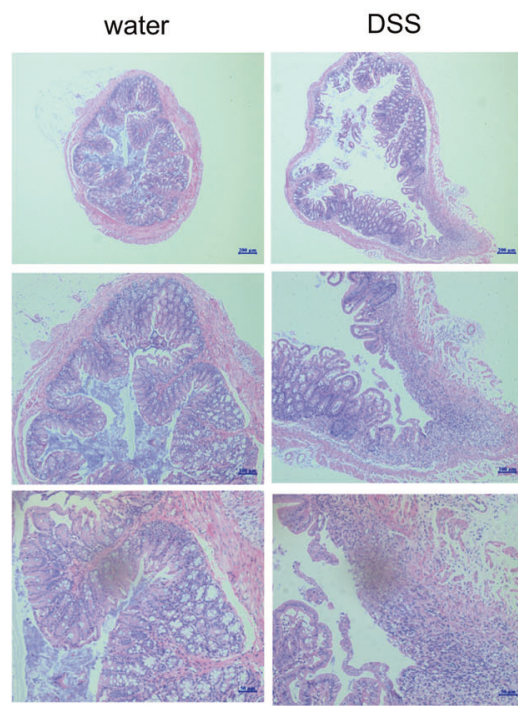

metformin-1

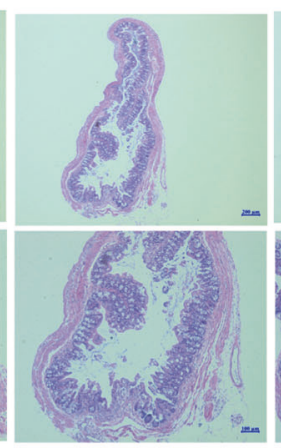

B

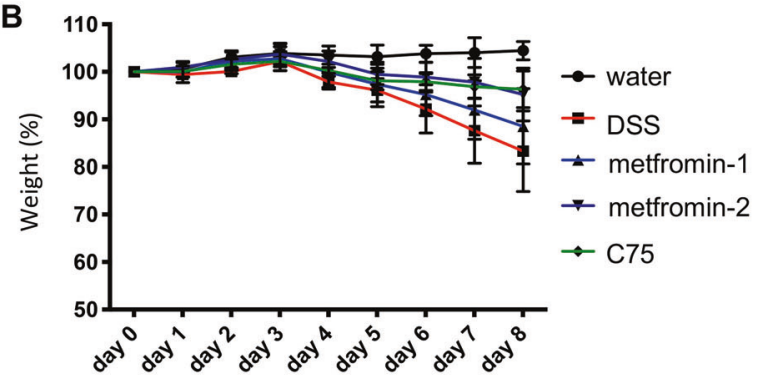

D

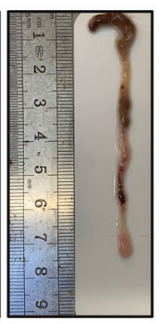

$c^{15}$

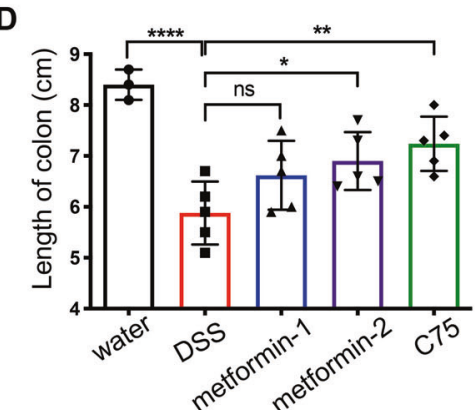

F

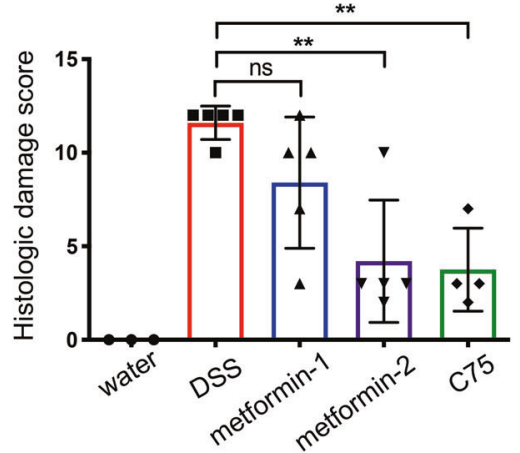

metformin-2

C75
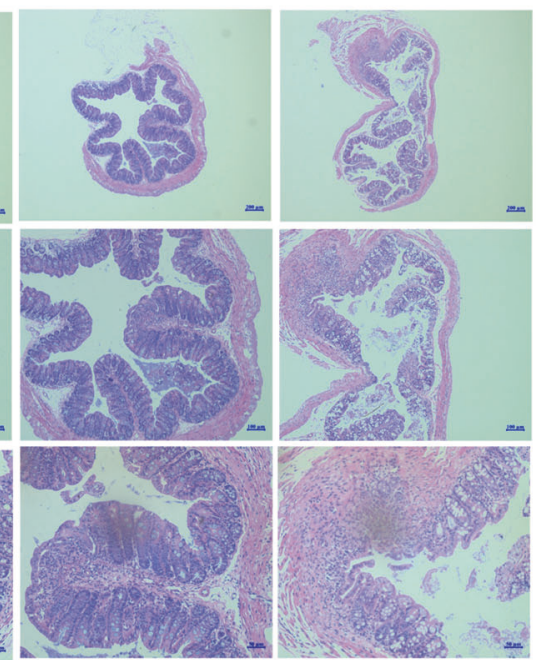

Fig. 7 The therapeutic effect of metformin and C75 on dextran sulfate sodium (DSS)-induced colitis mice. A An overview of animal experiments (five mice in each group). B Weight loss was measured each day during the whole experiment. C, D After mice were sacrificed, the length of colons was recorded and quantified. E Disease activity index (DAl) was calculated according to weight loss and characteristics of feces. F, G Hematoxylin and eosin (H\&E) staining was utilized to evaluate colon damages. The extent of damages was quantified by a score ranging from 0 to 12 (F). Representative images of H\&E staining (G). metformin-1: $100 \mathrm{mg} / \mathrm{kg} ; \mathrm{metformin}-2: 200 \mathrm{mg} / \mathrm{kg} ; \mathrm{C75}: 5 \mathrm{mg} / \mathrm{kg}$. Data were represented as means \pm SD. ${ }^{*} p<0.05,{ }^{* *} p<0.01,{ }^{* * *} p<0.001,{ }^{* * * *} p<0.0001$, ANOVA. 
A
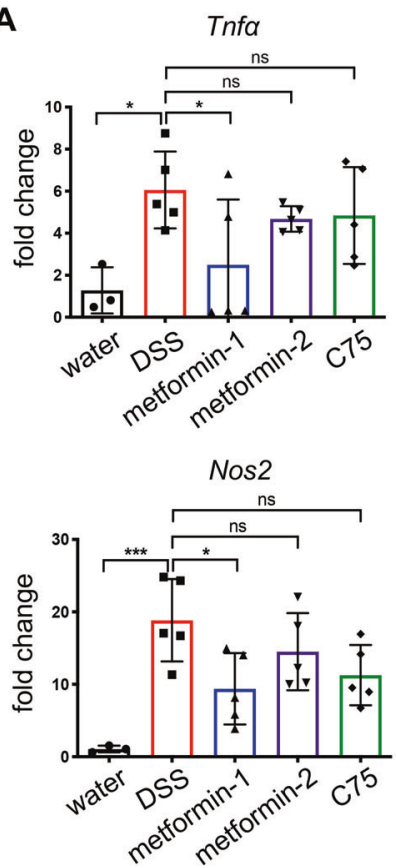

C

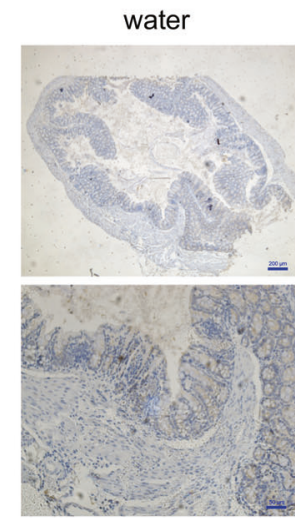

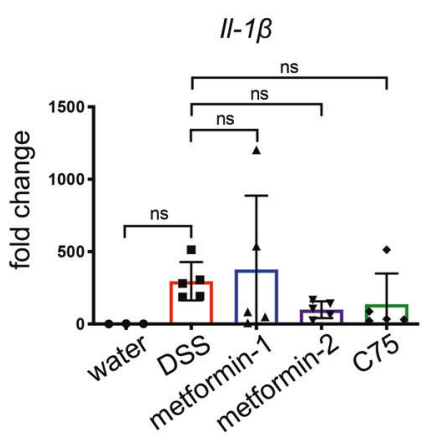
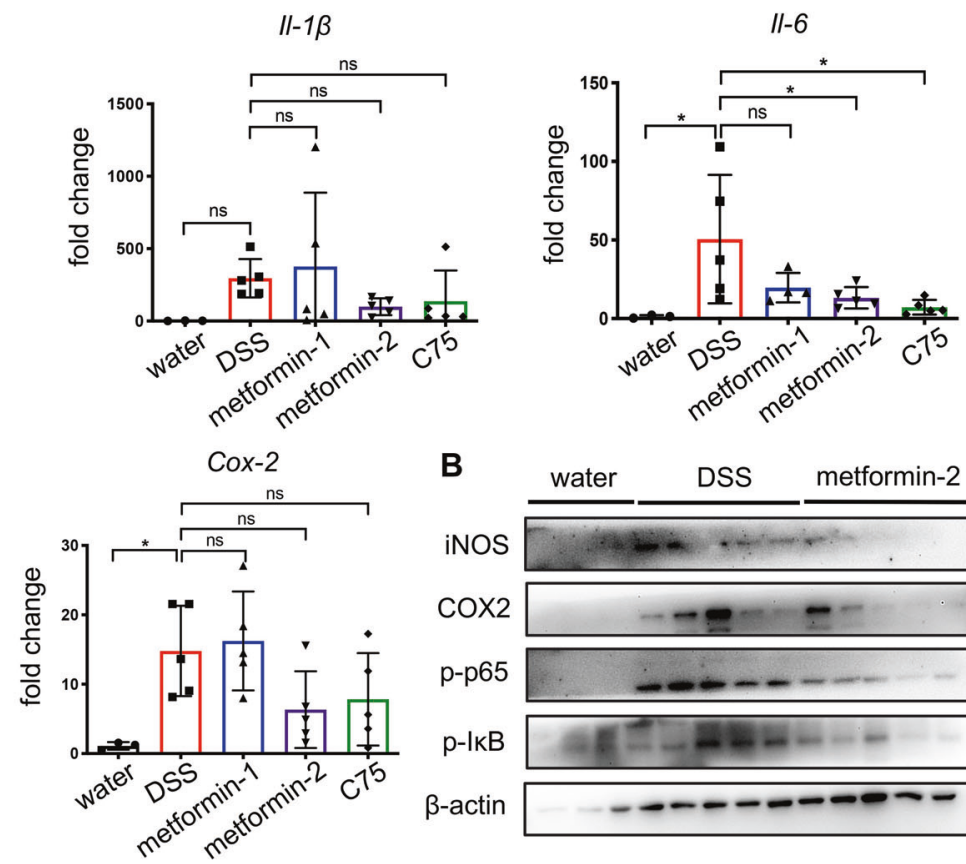

B

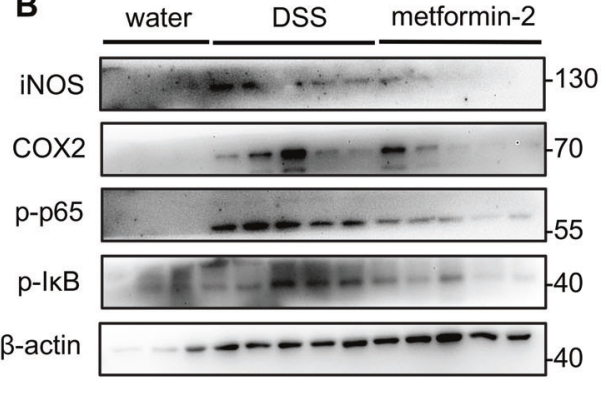

DSS

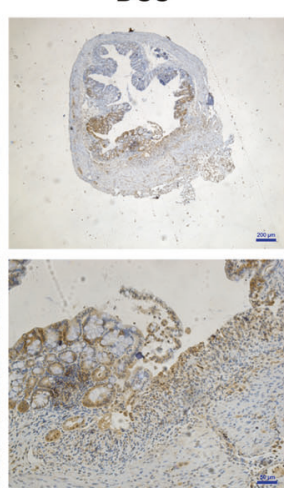

metformin-1

metformin-2
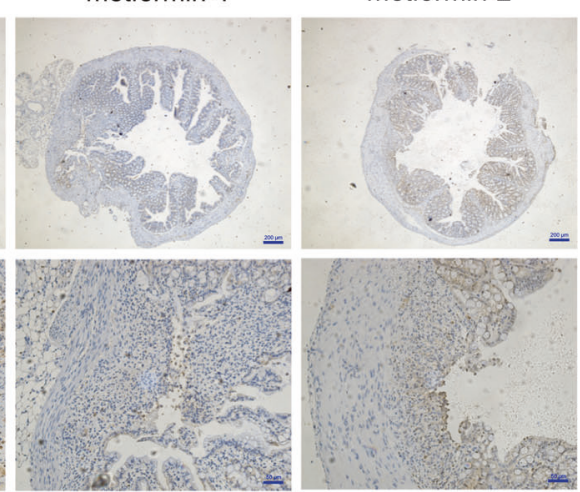

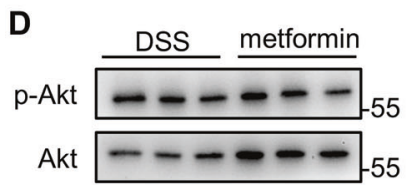

E

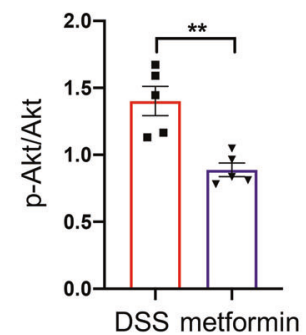

$\mathbf{F}$

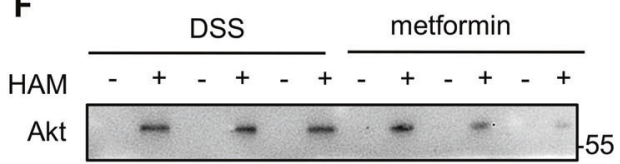

LPMCs IP: biotin

Akt

$\beta$-actin

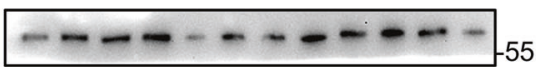

$---\infty-\cdots-\cdots-\infty$

LPMCs input
G

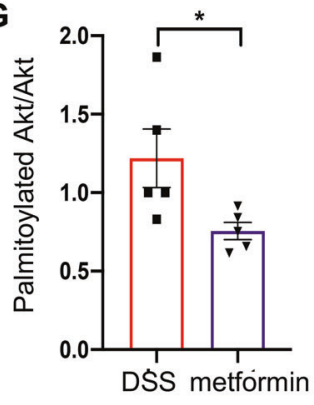

Fig. 8 Metformin suppressed proinflammatory responses of colonic LPMCs in DSS-induced colitis mice. Murine lamina propria mononuclear cells (LPMCs) were isolated from colons on day 8. A The mRNA levels of Tnfa, II-1 $\beta$, II-6, Cox-2, and Nos2 in LPMCs were determined by qRT-PCR. B Proinflammatory proteins in LPMCs were determined by Western blotting. C The expression of TNF $\alpha$ in colons was detected by immunohistochemistry. D, E The phosphorylation of Akt was inhibited by metformin in colonic LPMCs. Representative images of western blotting (D) and quantification of p-Akt/Akt ratios (E). F, G The palmitoylation of Akt in colonic LPMCs was detected by the ABE method. Representative images of western blotting (F) and quantification of palmitoylated Akt/Akt ratios (G). Data were represented as means \pm SD. ${ }^{*} p<0.05,{ }^{* *} p<0.01,{ }^{* * *} p<0.001,{ }^{* * * *} p<0.0001$, ANOVA or $t$-test. 
indispensable for LPS-induced proinflammatory responses in macrophages.

To be more detailed, we identified that the LPS-activated Akt/ mTOR pathway was hindered by metformin or FASN inhibition in macrophages. It has been well known that membrane recruitment is a major requirement for Akt activation [40]. Since Akt primarily exists in the cytosol, there undoubtedly occurs some unclear molecule events which assist it to approach to plasma membrane. The immunofluorescence and western blotting experiments exhibited that metformin as well as FASN inhibition disrupted the membrane localization of Akt, which guided us to further investigate the probable mechanism of Akt trafficking. A previous report identified that TRAF6, as a E3 ligase promoting Akt ubiquitinated, is crucial for IGF-1-induced Akt membrane distribution and phosphorylation at T308 and S473 in MEFs [53]. With K63ubiquitination contributing to Akt trafficking, we were interested in the correlation between its post-translational modification and membrane localization. Palmitic acid synthesized by FASN can participate in a protein post-translational modification, called $S$ palmitoylation. S-palmitoylation belongs to lipidation [13, 14]. De novo fatty acid synthesis has been validated to contribute to myeloid differentiation primary response protein (MYD88) palmitoylation, which is a major requirement for IRAK4 binding and downstream signal activation [11]. In addition, we were inspired by another fact that palmitoylation-mediated hydrophobicity facilitates protein attaching to membranes. In our study, we identified a previously undescribed S-palmitoylated protein, Akt, whose palmitoylation was dependent on FASN. No matter knockdown or pharmacological inhibition of FASN, Akt palmitoylation was reduced. Further, we connected the downregulated palmitoylated Akt with its suppressed membrane localization through confirming the influence of a palmitoyltransferase inhibitor, 2-BP, on Akt distribution and activation. Consequently, the specific mechanism of how metformin regulated Akt activation was to modulate FASN-dependent palmitoylation of Akt.

It is generally recognized that Akt as an upstream regulator increases activity of the transcription factor SREBP-1, resulting in an elevation in FASN mRNA expression [54]. In normal physiological conditions, insulin-responsive cells exposed to insulin, such as adipocytes and hepatocytes, undergo metabolism changes. The activated insulin receptor initiates PI3K/Akt signaling, which further facilitates glycogen and fatty acid synthesis through regulating GSK3 $\beta$ and mTORC1 [55-57]. On the other hand, a number of cancer-associated reports shows that PI3K signaling modulates FASN levels as well in cancerous cells [58-60]. Conversely, it is also verified that FASN acts upstream of Akt and inhibition of FASN downregulates phosphorylated Akt, though the specific mechanism remains unclear $[37,38]$. Indeed, our study provided some evidence on the specific mechanism that FASN as a metabolic enzyme regulated palmitoylation-dependent activation of Akt. On the basis of positive self-amplifying feedback between Akt activation and FASN, we speculated that it was meaningful and effective to break the abnormally hyperactive feedback cycle in pathological conditions.

Numerous studies confirmed that metformin had a direct inhibitory effect on proinflammatory responses of macrophages, generally through AMPK activation or inhibiting mitochondrial reactive oxygen species (ROS) production [21-23]. The immunomodulatory properties of metformin have also been suggested to underlie some of its beneficial effects on autoimmune inflammatory diseases [30,61, 62]. Though metformin is well known as a popular drug regulating cellular metabolism, the contribution of metformin-induced changes in cellular metabolism to its antiinflammatory function remains unknown. It was significant for our work to be the first to demonstrate that the anti-inflammatory effect of metformin was, at least partly, attributed to inhibiting fatty acid synthesis metabolism in macrophages. Similar with C75, metformin reduced fatty acid synthesis and compromised Akt palmitoylation in LPS-stimulated macrophages, further disrupting its membrane recruitment and phosphorylation. Inactivation of Akt resulted in less phosphorylation of p65 in the end. In brief, we succeeded in uncovering the anti-inflammatory mechanism of metformin from the immune metabolism perspective.

S-palmitoylation reactions are directly catalyzed by a family of aspartate-histidine-histidine-cysteine palmitoyl acyl transferases (DHHC-PATs) [14]. Therefore, we plan to further investigate the exact DHHC-PATs mediating Akt palmitoylation in a subsequent study.

\section{CONCLUSIONS}

Collectively, it is demonstrated that metformin alleviates inflammation through inhibiting endogenous fatty acid synthesis and its related Akt palmitoylation in macrophages. Our data provides substantial evidence supporting FASN as a therapeutic target and metformin as a potential treatment for macrophages-mediated inflammatory diseases.

\section{DATA AVAILABILITY}

All data are available in the paper or supplementary materials.

\section{REFERENCES}

1. Shapouri-Moghaddam A, Mohammadian S, Vazini H, Taghadosi M, Esmaeili SA, Mardani $F$, et al. Macrophage plasticity, polarization, and function in health and disease. J Cell Physiol. 2018;233:6425-40.

2. O'Neill LA, Kishton RJ, Rathmell J. A guide to immunometabolism for immunologists. Nat Rev Immunol. 2016;16:553-65.

3. Pearce EL, Pearce EJ. Metabolic pathways in immune cell activation and quiescence. Immunity. 2013;38:633-43.

4. Saha S, Shalova IN, Biswas SK. Metabolic regulation of macrophage phenotype and function. Immunol Rev. 2017;280:102-11.

5. Van den Bossche J, O'Neill LA, Menon D. Macrophage Immunometabolism: where are we (going)? Trends Immunol. 2017;38:395-406.

6. Qian X, Yang Z, Mao E, Chen E. Regulation of fatty acid synthesis in immune cells. Scand J Immunol. 2018;88:e12713.

7. Tzeng HT, Chyuan IT, Chen WY. Shaping of innate immune response by fatty acid metabolite palmitate. Cells. 2019;8:1633.

8. Huang S, Rutkowsky JM, Snodgrass RG, Ono-Moore KD, Schneider DA, Newman $J W$, et al. Saturated fatty acids activate TLR-mediated proinflammatory signaling pathways. J Lipid Res. 2012;53:2002-13.

9. Ecker J, Liebisch G, Englmaier M, Grandl M, Robenek H, Schmitz G. Induction of fatty acid synthesis is a key requirement for phagocytic differentiation of human monocytes. Proc Natl Acad Sci USA. 2010;107:7817-22.

10. Wei $X$, Song H, Yin L, Rizzo MG, Sidhu R, Covey DF, et al. Fatty acid synthesis configures the plasma membrane for inflammation in diabetes. Nature. 2016;539:294-8.

11. Kim YC, Lee SE, Kim SK, Jang HD, Hwang I, Jin S, et al. Toll-like receptor mediated inflammation requires FASN-dependent MYD88 palmitoylation. Nat Chem Biol. 2019;15:907-16.

12. Wei X, Yang Z, Rey FE, Ridaura VK, Davidson NO, Gordon Jl, et al. Fatty acid synthase modulates intestinal barrier function through palmitoylation of mucin 2 . Cell Host Microbe. 2012;11:140-52.

13. De I, Sadhukhan S. Emerging roles of DHHC-mediated protein S-palmitoylation in physiological and pathophysiological context. Eur J Cell Biol. 2018;97:319-38.

14. Greaves J, Chamberlain LH. DHHC palmitoyl transferases: substrate interactions and (patho)physiology. Trends Biochem Sci. 2011;36:245-53.

15. Lu Y, Zheng Y, Coyaud E, Zhang C, Selvabaskaran A, Yu Y, et al. Palmitoylation of NOD1 and NOD2 is required for bacterial sensing. Science. 2019;366:460-7.

16. Abdel-Wahab AF, Mahmoud W, Al-Harizy RM. Targeting glucose metabolism to suppress cancer progression: prospective of anti-glycolytic cancer therapy. Pharm Res. 2019;150:104511.

17. Foretz M, Guigas B, Viollet B. Understanding the glucoregulatory mechanisms of metformin in type 2 diabetes mellitus. Nat Rev Endocrinol. 2019;15:569-89.

18. Zhou J, Massey S, Story D, Li L. Metformin: an old drug with new applications. Int J Mol Sci. 2018;19:2863.

19. Jenkins $A J$, Welsh $P$, Petrie JR. Metformin, lipids and atherosclerosis prevention. Curr Opin Lipido. 2018;29:346-53. 
20. Mazza A, Fruci B, Garinis GA, Giuliano S, Malaguarnera R, Belfiore A. The role of metformin in the management of NAFLD. Exp Diabetes Res. 2012;2012:716404.

21. Vasamsetti SB, Karnewar S, Kanugula AK, Thatipalli AR, Kumar JM, Kotamraju S. Metformin inhibits monocyte-to-macrophage differentiation via AMPK-mediated inhibition of STAT3 activation: potential role in atherosclerosis. Diabetes. 2015;64:2028-41.

22. Kim J, Kwak HJ, Cha JY, Jeong YS, Rhee SD, Kim KR, et al. Metformin suppresses lipopolysaccharide (LPS)-induced inflammatory response in murine macrophages via activating transcription factor-3 (ATF-3) induction. J Biol Chem. 2014;289:23246-55.

23. Kelly B, Tannahill GM, Murphy MP, O'Neill LA. Metformin inhibits the production of reactive oxygen species from $\mathrm{NADH}$ : ubiquinone oxidoreductase to limit induction of interleukin-1beta (IL-1beta) and boosts interleukin-10 (IL-10) in lipopolysaccharide (LPS)-activated macrophages. J Biol Chem. 2015;290:20348-59.

24. Toda G, Yamauchi T, Kadowaki T, Ueki K. Preparation and culture of bone marrowderived macrophages from mice for functional analysis. STAR Protoc. 2021;2:100246.

25. Wan J, Roth AF, Bailey AO, Davis NG. Palmitoylated proteins: purification and identification. Nat Protoc. 2007;2:1573-84.

26. Brigidi GS, Bamji SX. Detection of protein palmitoylation in cultured hippocampal neurons by immunoprecipitation and acyl-biotin exchange (ABE). J Vis Exp. 2013; 72:e50031.

27. Chassaing B, Aitken JD, Malleshappa M, Vijay-Kumar M. Dextran sulfate sodium (DSS)-induced colitis in mice. Curr Protoc Immunol. 2014;104:1525.1-1525.14.

28. Di Fusco D, Dinallo V, Monteleone I, Laudisi F, Marafini I, Franze E, et al. Metformin inhibits inflammatory signals in the gut by controlling AMPK and p38 MAP kinase activation. Clin Sci. 2018;132:1155-68.

29. Matsuo S, Yang WL, Aziz M, Kameoka S, Wang P. Fatty acid synthase inhibitor C75 ameliorates experimental colitis. Mol Med. 2014;20:1-9.

30. Lee SY, Lee SH, Yang EJ, Kim EK, Kim JK, Shin DY, et al. Metformin ameliorates inflammatory bowel disease by suppression of the STAT3 signaling pathway and regulation of the between Th17/Treg balance. PLoS ONE. 2015;10:e0135858.

31. Weigmann B, Tubbe I, Seidel D, Nicolaev A, Becker C, Neurath MF. Isolation and subsequent analysis of murine lamina propria mononuclear cells from colonic tissue. Nat Protoc. 2007;2:2307-11.

32. Menendez JA, Lupu R. Fatty acid synthase and the lipogenic phenotype in cancer pathogenesis. Nat Rev Cancer. 2007;7:763-77.

33. Dinarello CA. Proinflammatory cytokines. Chest. 2000;118:503-8.

34. Guha M, Mackman N. LPS induction of gene expression in human monocytes. Cell Signal. 2001;13:85-94.

35. Zhu X, Yan H, Xia M, Chang X, Xu X, Wang L, et al. Metformin attenuates triglyceride accumulation in HepG2 cells through decreasing stearyl-coenzyme $A$ desaturase 1 expression. Lipids Health Dis. 2018;17:114.

36. Zhao Y, Hu X, Liu Y, Dong S, Wen Z, He W, et al. ROS signaling under metabolic stress: cross-talk between AMPK and AKT pathway. Mol Cancer. 2017;16:79.

37. Wang HQ, Altomare DA, Skele KL, Poulikakos PI, Kuhajda FP, Di Cristofano A, et al. Positive feedback regulation between AKT activation and fatty acid synthase expression in ovarian carcinoma cells. Oncogene. 2005;24:3574-82.

38. Wagner R, Stubiger G, Veigel D, Wuczkowski M, Lanzerstorfer P, Weghuber J, et al. Multi-level suppression of receptor-PI3K-mTORC1 by fatty acid synthase inhibitors is crucial for their efficacy against ovarian cancer cells. Oncotarget. 2017;8:11600-13.

39. Alessi DR, James SR, Downes $C P$, Holmes AB, Gaffney PR, Reese $C B$, et al. Characterization of a 3-phosphoinositide-dependent protein kinase which phosphorylates and activates protein kinase Balpha. Curr Biol. 1997;7:261-9.

40. Bellacosa A, Chan TO, Ahmed NN, Datta K, Malstrom S, Stokoe D, et al. Akt activation by growth factors is a multiple-step process: the role of the $\mathrm{PH}$ domain. Oncogene. 1998;17:313-25.

41. Ebner M, Lucic I, Leonard TA, Yudushkin I. PI(3,4,5)P3 engagement restricts akt activity to cellular membranes. Mol Cell. 2017;65:416-31.e416.

42. Ning W, Jiang P, Guo Y, Wang C, Tan X, Zhang W, et al. GPS-Palm: a deep learningbased graphic presentation system for the prediction of S-palmitoylation sites in proteins. Brief Bioinform. 2020;22:1836-47.

43. Kaminska B. MAPK signalling pathways as molecular targets for antiinflammatory therapy-from molecular mechanisms to therapeutic benefits. Biochim Biophys Acta. 2005;1754:253-62.

44. Wirtz S, Popp V, Kindermann M, Gerlach K, Weigmann B, Fichtner-Feigl S, et al. Chemically induced mouse models of acute and chronic intestinal inflammation. Nat Protoc. 2017;12:1295-309.

45. Jones SF, Infante JR. Molecular pathways: fatty acid synthase. Clin Cancer Res. 2015;21:5434-8.

46. Yang CS, Matsuura K, Huang NJ, Robeson AC, Huang B, Zhang L, et al. Fatty acid synthase inhibition engages a novel caspase-2 regulatory mechanism to induce ovarian cancer cell death. Oncogene. 2015;34:3264-72.

47. Menendez JA, Lupu R. Fatty acid synthase (FASN) as a therapeutic target in breast cancer. Expert Opin Ther Targets. 2017;21:1001-16.
48. Ali A, Levantini E, Teo JT, Goggi J, Clohessy JG, Wu CS, et al. Fatty acid synthase mediates EGFR palmitoylation in EGFR mutated non-small cell lung cancer. EMBO Mol Med. 2018;10:e8313.

49. Zadra G, Ribeiro CF, Chetta P, Ho Y, Cacciatore S, Gao X, et al. Inhibition of de novo lipogenesis targets androgen receptor signaling in castration-resistant prostate cancer. Proc Natl Acad Sci USA. 2019;116:631-40.

50. Berod L, Friedrich C, Nandan A, Freitag J, Hagemann S, Harmrolfs K, et al. De novo fatty acid synthesis controls the fate between regulatory $T$ and $T$ helper 17 cells. Nat Med. 2014;20:1327-33.

51. Young KE, Flaherty S, Woodman KM, Sharma-Walia N, Reynolds JM. Fatty acid synthase regulates the pathogenicity of Th17 cells. J Leukoc Biol. 2017;102:1229-35.

52. Moon JS, Lee S, Park MA, Siempos II, Haslip M, Lee PJ, et al. UCP2-induced fatty acid synthase promotes NLRP3 inflammasome activation during sepsis. J Clin Invest. 2015;125:665-80.

53. Yang WL, Wang J, Chan $\mathrm{CH}$, Lee SW, Campos AD, Lamothe $B$, et al. The E3 ligase TRAF6 regulates Akt ubiquitination and activation. Science. 2009;325:1134-8.

54. Porstmann T, Santos CR, Griffiths B, Cully M, Wu M, Leevers S, et al. SREBP activity is regulated by mTORC1 and contributes to Akt-dependent cell growth. Cell Metab. 2008;8:224-36.

55. Deng Q, Ma D, Sun G, Yuan X, Wang Z, Liu G. PTEN influences insulin and lipid metabolism in bovine hepatocytes in vitro. J Dairy Res. 2019;86:73-76.

56. Radenne A, Akpa M, Martel C, Sawadogo S, Mauvoisin D, Mounier C. Hepatic regulation of fatty acid synthase by insulin and T3: evidence for T3 genomic and nongenomic actions. Am J Physiol Endocrinol Metab. 2008;295:E884-894.

57. Schultze SM, Hemmings BA, Niessen M, Tschopp O. PI3K/AKT, MAPK and AMPK signalling: protein kinases in glucose homeostasis. Expert Rev Mol Med. 2012;14:e1.

58. Zhao J, Zhang X, Gao T, Wang S, Hou Y, Yuan P, et al. SIK2 enhances synthesis of fatty acid and cholesterol in ovarian cancer cells and tumor growth through PI3K/ Akt signaling pathway. Cell Death Dis. 2020;11:25.

59. Van de Sande T, De Schrijver E, Heyns W, Verhoeven G, Swinnen JV. Role of the phosphatidylinositol 3'-kinase/PTEN/Akt kinase pathway in the overexpression of fatty acid synthase in LNCaP prostate cancer cells. Cancer Res. 2002;62:642-6.

60. Yang YA, Han WF, Morin PJ, Chrest FJ, Pizer ES. Activation of fatty acid synthesis during neoplastic transformation: role of mitogen-activated protein kinase and phosphatidylinositol 3-kinase. Exp Cell Res. 2002;279:80-90.

61. Son HJ, Lee J, Lee SY, Kim EK, Park MJ, Kim KW, et al. Metformin attenuates experimental autoimmune arthritis through reciprocal regulation of Th17/Treg balance and osteoclastogenesis. Mediators Inflamm. 2014;2014:973986.

62. Nath N, Khan M, Paintlia MK, Singh I, Hoda MN, Giri S. Metformin attenuated the autoimmune disease of the central nervous system in animal models of multiple sclerosis. J Immunol. 2009;182:8005-14.

\section{AUTHOR CONTRIBUTIONS}

$X$ Zou and WX conceived and devised the project. $X$ Zhang and $Y Z$ coordinated the project. WX and KS conducted the experiments with the assistance of YZ. WX and KS analyzed the statistics and interpreted the results. WX wrote the manuscript. $X Z$ Zou, $X$ Zhang, and $Y Z$ revised the manuscript. $X$ Zou, $X$ Zhang, and $Y Z$ supervised the work. The final article was approved by all of the authors.

\section{FUNDING}

This study was supported by the National Natural Science Foundation of China (No.81871947) and Six Talent Peaks Project of Jiangsu Province (WSW-134).

\section{COMPETING INTERESTS}

The authors declare no competing interests.

\section{ADDITIONAL INFORMATION}

Supplementary information The online version contains supplementary material available at https://doi.org/10.1038/s41419-021-04235-0.

Correspondence and requests for materials should be addressed to Xiaoqi Zhang, Yi-Hua Zhou or Xiaoping Zou.

Reprints and permission information is available at http://www.nature.com/ reprints

Publisher's note Springer Nature remains neutral with regard to jurisdictional claims in published maps and institutional affiliations. 
Open Access This article is licensed under a Creative Commons At c) Atribution 4.0 International License, which permits use, sharing,
adaptation, distribution and reproduction in any medium or format, as long as you give appropriate credit to the original author(s) and the source, provide a link to the Creative Commons license, and indicate if changes were made. The images or other third party material in this article are included in the article's Creative Commons license, unless indicated otherwise in a credit line to the material. If material is not included in the article's Creative Commons license and your intended use is not permitted by statutory regulation or exceeds the permitted use, you will need to obtain permission directly from the copyright holder. To view a copy of this license, visit http://creativecommons. org/licenses/by/4.0/.

(c) The Author(s) 2021 